\title{
Microfoams of Biopolymers by Laser-Induced Stretching: Mechanisms and Applications
}

\author{
Lazare Sylvain \\ Institut des Sciences Moléculaires (ISM) UMR 5255, Université Bordeaux 1 \\ 351 cours de la Libération, 33405 Talence \\ France
}

\section{Introduction}

Biopolymers and polymers derived from the biomass (Gandini, 2008; Mooney, 2009) are abundant natural chemical substances that find applications in many fields like agriculture, agro-food industry, textile and medicine, etc.. . A vast majority of them does not cause problems to the environment (Pérez et al., 2002), contrary to synthetic polymers (Moore, 2008). Their natural resource is continuously renewed and they are traditionally used since the prehistoric times. The recent problems like climate change, fossil fuels depletion and growing costs of recycling due to strictly observed modern environmental concerns, have renewed our interest for a modern form of bio-based economy. This results in an increased use of natural substances and a search for new energy efficient engineering processes. The laser-based technologies have emerged (Bäuerle, 2000) since the early sixties date of the laser birth, that is to say very recently, and are now explored for new types of processing, like micro-precise laser characterization, ablation and machining, by various appealing experimental approaches (Fig.1). Laser processing and characterization of materials in general offer definite advantages like the cost-efficiency due to rapidity and the submicroprecision (Lazare \& Tokarev 2005a). They are considered as techniques lending themselves easily to future automation and industrialization. Moreover it is generally recognized that laser beams make it possible to perform a remote characterization and treatment of the target with a large working distance, which in the extreme case can easily be up to the kilometer range (as in LIDAR) depending on the laser beam quality (Telle et al., 2007). A new micro-foaming transition of biopolymer films was discovered (Lazare et al., 2005b) during the study of laser ablation of collagen, a biopolymer extracted from rat tails tendon. In these experiments the absorption of one $\mathrm{KrF}$ excimer laser pulse produces a layer of foamy material on the surface of the collagen film (Fig.1). For the first time, with a unique pulse a clearly visible and highly interesting transformation of a material was produced, and which was not the simple removal of matter like in regular laser ablation (Figs.1.1 for surface ablation, 1.2 for multi-pulse micro-drilling, and 1.3 for surface modification), often called "clean ablation" as known from previous studies (Weisbuch, 1999; Tokarev, 2003; Lippert \& Dickinson, 2003; Elaboudi, 2008). Usually thermal and photochemical decomposition of the polymer are considered to account for the ejection of the gas products. 
In the foaming process, whose mechanism has been studied and modeled (Lazare, 2009) since the discovery, a more selective combination of laser-induced forces is involved to explain the sudden expansion of the laser-excited polymer. This expansion approximatively occurs in a timescale of the order of $50 \mathrm{~ns}$ after the end the laser pulse, when the high pressure wave induced by the laser excitation energy propagates away from the surface and gives rise to a tensile wave. It results in a sudden and dense cavitation phenomenon yielding a micro-foam composed of closed or open microscopic cells, quite similarly to the chemically engineered polymer foams, although the timescale is comparatively quite smaller. It is shown that, for the laser-induced foaming to occur, the target polymer must be moderately absorbing in order to generate a strong pressure wave and the laser absorption must provide enough gas pressure produced by the ablation products. Biopolymers tend to display coefficients of absorption situated in the adequate range centered around $\sim 10^{3} \mathrm{~cm}^{-1}$. The obtained microfoams of biopolymers display micro-features, microscopic cells and submicron-size filaments, having some similarities with that of familiar biomaterials e.g. wood, tissue matrix, etc.. . They are therefore expected to find applications in various fields of biomaterial sciences e.g. in biomedical. The purposes of this paper are mainly to review the results, to discuss the mechanisms and speculate about the future applications. Among the laser ablation regimes studied up to now the photomechanical regime, which allows ablation by using high pressure rather than high temperature, is still recent and mostly unknown. The main questions to be answered are 1) how matter is moved from the surface, by which mechanisms 2) is part of the surface material expulsed from the target and in which form, 3) is molecular decomposition necessary, to which extend the polymer is decomposed into gas molecules and 4) what are the laser and material parameters that best control the process and that can be adjusted to select the sub-regime of interest?. The experimental results and the explanatory theoretical modeling are presented in the following text.

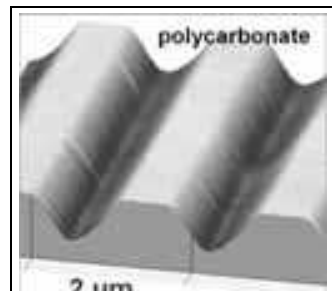

$2 \mu \mathrm{m}$
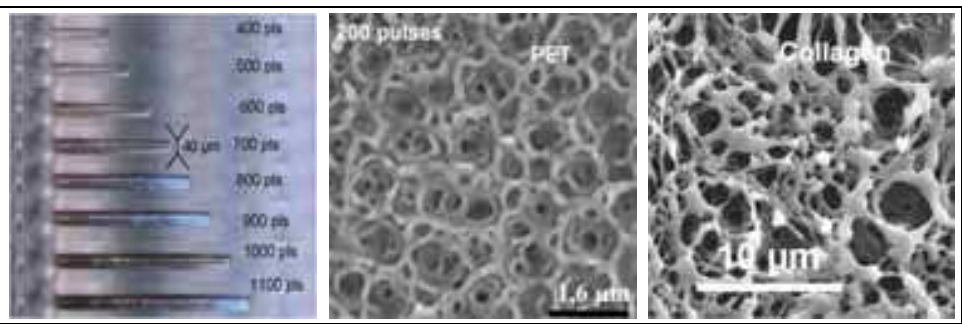

Fig. 1. Various laser (KrF laser radiation) processing of polymers studied in the recent years. Left to right: 1) Micro-precise surface ablation of polycarbonate (one pulse), 2) Micro-drilling in polycarbonate, 3) Surface modification of poly(ethylene terephthalate), 4) Micro-foam induced by a single laser pulse $\left(0.9 \mathrm{~J} / \mathrm{cm}^{2}\right)$ in the surface of a collagen film.

\section{Lasers systems}

Lasers used in this work are either nanosecond $\mathrm{KrF}$ ultraviolet lasers or ultrashort pulses femtosecond lasers for the time resolved experiments. $\mathrm{KrF}$ excimer lasers are classical excimer lasers which have been described in details in previous works. The $\mathrm{KrF}$ laser radiation at $248 \mathrm{~nm}$ is a good compromise since biopolymers are in general moderately absorbing at this wavelength. The main qualities are the large energy per pulse $(\sim 500 \mathrm{~mJ})$ 
and the long-lasting gas mixture $\left(\mathrm{Kr} / \mathrm{F}_{2} / \mathrm{Ne}\right)$ filling which makes it cost efficient. The beam is patterned with homemade optical setups and attenuated variably and at constant spot area (either $0.1 \times 0.1 \mathrm{~mm}^{2}$ or $1.0 \times 1.0 \mathrm{~mm}^{2}$ ) in order to control over a large range the energy density $\left(0.001\right.$ to $\left.\sim 12 \mathrm{~J} / \mathrm{cm}^{2}\right)$ delivered to the target. The energy profile on the spot area was near homogeneous in all cases, or measured as approximately Gaussian. The energy of each pulse is carefully monitored with a detector, a precaution made necessary by the singlepulse experiments. The femtosecond laser system has a pulse width of 90 fs (Gaspard, 2008a) and is composed of a Ti:sapphire amplifier delivering linearly polarized fs pulses centered at $800 \mathrm{~nm}$ at a maximum repetition rate of $1 \mathrm{kHz}$. For some experiments isolation of single pulses was carried out by a Pockels cell in the regenerative cavity. Wavelength of the final pulse could be tuned by frequency conversion to 2 and $3 \omega$ (resp. 400 and $266 \mathrm{~nm}$ ) with BBO crystals. The absorption mechanisms of the femtosecond pulses are different from the 1 photon-induced electronic transition involved in the ultraviolet nanosecond laser interaction. More multiphoton type of electronic excitation takes place in the fs case and makes it possible to yield ablation with materials that cannot be excited by nanosecond laser pulse due to the absence of absorption.

\section{Materials}

\subsection{Biopolymers of Interest}

It happens that most biopolymers have optical features that are well suited to obtain the present laser-induced foaming phenomena. First they must have a moderate absorption coefficient, approximately $\alpha<3000 \mathrm{~cm}^{-1}$, in order to generate a strong pressure wave. Second enough gas pressure must be produced during their ablation in order to support the cavitation phenomenon that occurs upon the laser-induced stretching. Third their viscosity must decrease rapidly upon the laser heating (low $T_{\mathrm{g}}$ ) for providing a high nucleation rate giving rise to a dense cavitation and bubbling. If these conditions are met at the same time, foaming is produced when one pulse is absorbed at the surface.

Polymers made with the glucose repeat unit obtained by the photosynthesis are the most abundantly produced by the biomass. Depending on the linkage between the glucose unit ( $\alpha$ or $\beta$ ), cellulose or starch molecules are obtained. The two molecules have different properties. Cellulose is a straight and rigid molecule maintained in a linear configuration by the side groups $\left(-\mathrm{CH}_{2} \mathrm{OH}\right)$ and the network of hydrogen bonds as in Fig.3 (Pinkert et al., 2009). It is linked to the neighbour cellulose molecules in a very stable way, so that elaborated 3D structures known in plants can be formed. In starch molecules the glucose repeat units are linked differently and the side groups are evenly distributed on the same side. Their steric effect induces a curvature in the polymer backbone which results in the formation of helical molecules. Because of this, starch molecules cannot be so strongly selfassociated than cellulose molecules.

Owing to the high insolubility of cellulose in common solvents (Liu et al., 2007), many derivatives have been studied for making it more industrially tractable. Some industrially important cellulose derivatives, like cellulose triacetate (CTA), cellulose acetate phthalate (CAP), cellulose dinitrate (CDN) (Fig.2), have been developed long ago but have been since then supplanted by synthetic polymers made by the petrochemistry. However they are regaining interest as polymers derived from sustainable resources. Other important biopolymers are proteinic molecules made of amino-acid chains. They are structurally very 


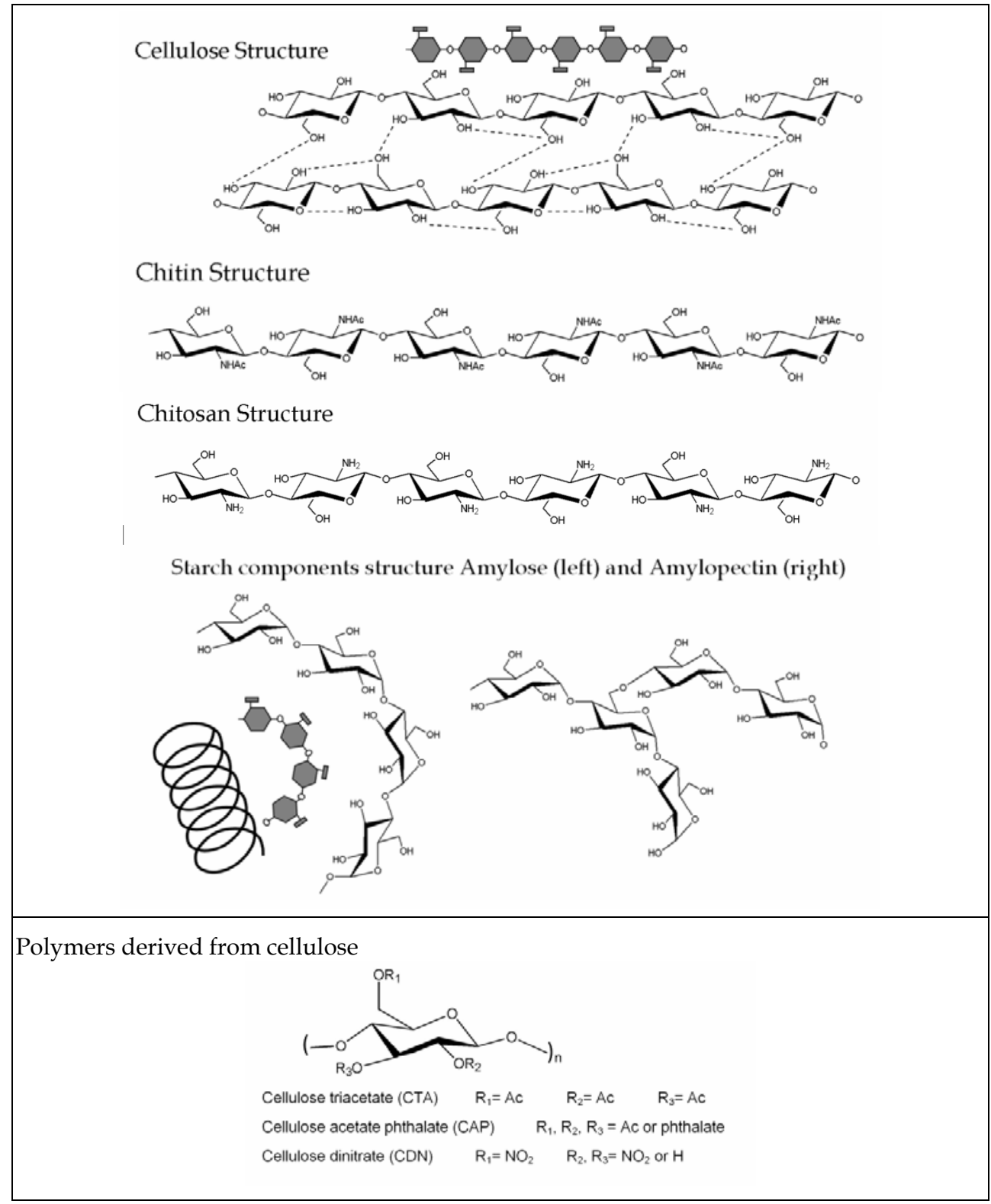

Fig. 2. Natural polymers studied in this work are based on glucose repeat unit (cellulose) or are protein type like collagen which is made of glycine, proline and hydroxyproline mainly. Cellulose is transformed into chitin by animals (insects, crustaceans) and mushrooms which is industrially derived in more tractable chitosan. Other industrially important cellulose derivatives include cellulose triacetate (CTA), cellulose acetate phthalate (CAP), cellulose dinitrate $(\mathrm{CDN})$. 
diverse and display a variety of molecular functions in natural systems as well. They are for a long time used as commodity polymers. Some of the most important practical interest include gelatin, casein, keratin, etc.. Here we present collagen (Gordon, 2010) from which gelatine is extracted. Collagen single strand is a polymer mostly composed of a repeating tripeptide (proline, hydroxyproline, glycine POG) which provides the capability of forming a triple helix by the winding of three strands (Fig.3). Triplet molecules can in turn selfassemble into fibrils and fibers which are integrated into the extracellular matrix. Apart being a structural material for cellular tissues the collagen network has a variety of functions like the presence of cryptic RGD (Arg-Gly-Asp)-containing peptides (Taubenberger et al., 2010) which can eventually be recognised by osteoblast cells in damaged tissue in need of reconstruction. Therefore collagen molecules are always engaged in a molecular assembly unless chemically treated by heat or aqueous basic solution. In that case the triple helix unwinds to yield gelatine, a random coil polymer having same amino-acid composition. It is the result of the so-called "melting" of the collagen assemblies.

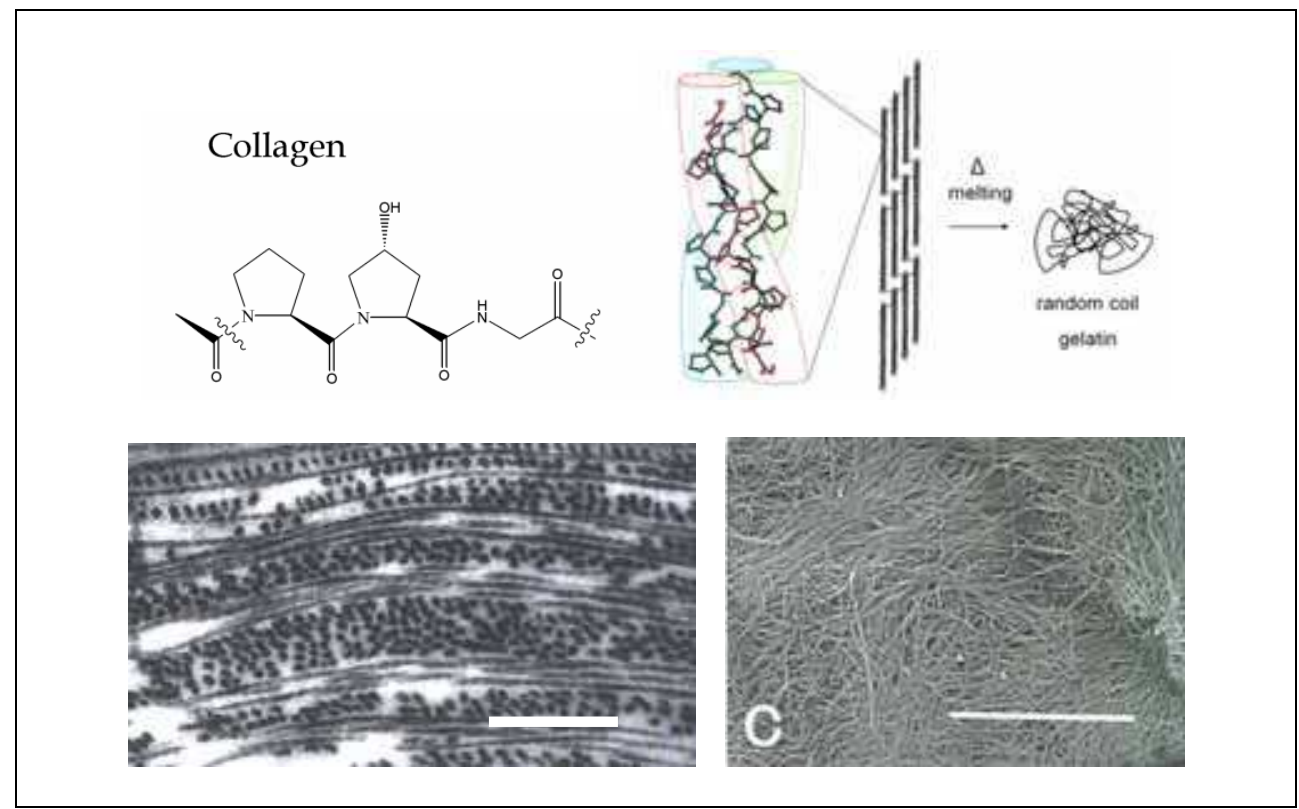

Fig. 3. Collagen single strand has a repeat unit mainly composed of tripeptide (proline, hydroxyproline and glycine POG). It is a structural protein whose molecule is a triple helix capable of easy self assembling. It is present in the form of fibrils in many tissues like cornea (picture on the left, scale bar is $1 \mu \mathrm{m}$ ) or lungs (picture on the right, scale bar is $100 \mu \mathrm{m}$ ). Collagen can be extracted in the triple helix form or can be denatured into a random coil form called gelatine under the action of a basic solution or hot water.

As mentioned previously and demonstrated below by the model, a polymer is a good candidate to laser-induced foaming if its absorption at the laser wavelength places it in the situation of pressure confinement, that is to say $\frac{l_{p}}{l_{\alpha}}=\frac{c_{s} \tau}{\alpha^{-1}}=\alpha c_{s} \tau<1$. Pressure propagation 
length $l_{\mathrm{p}}=c_{\mathrm{s}} \tau$, during the laser pulse duration $\tau$ must be much smaller than the laser absorption length $l_{\mathrm{a}}=1 / \alpha$ in order to observe an elevation of pressure. The model shows that the optimum of absorption coefficient is $1000 \mathrm{~cm}^{-1}$ if the assumed speed of sound is $1 \mu \mathrm{m} / \mathrm{s}$. Further short pulses favour pressure elevation and changing to smaller coefficients polymer necessitates increasing incident fluence to yield same elevation. The pressure model below brings more precise explanation.

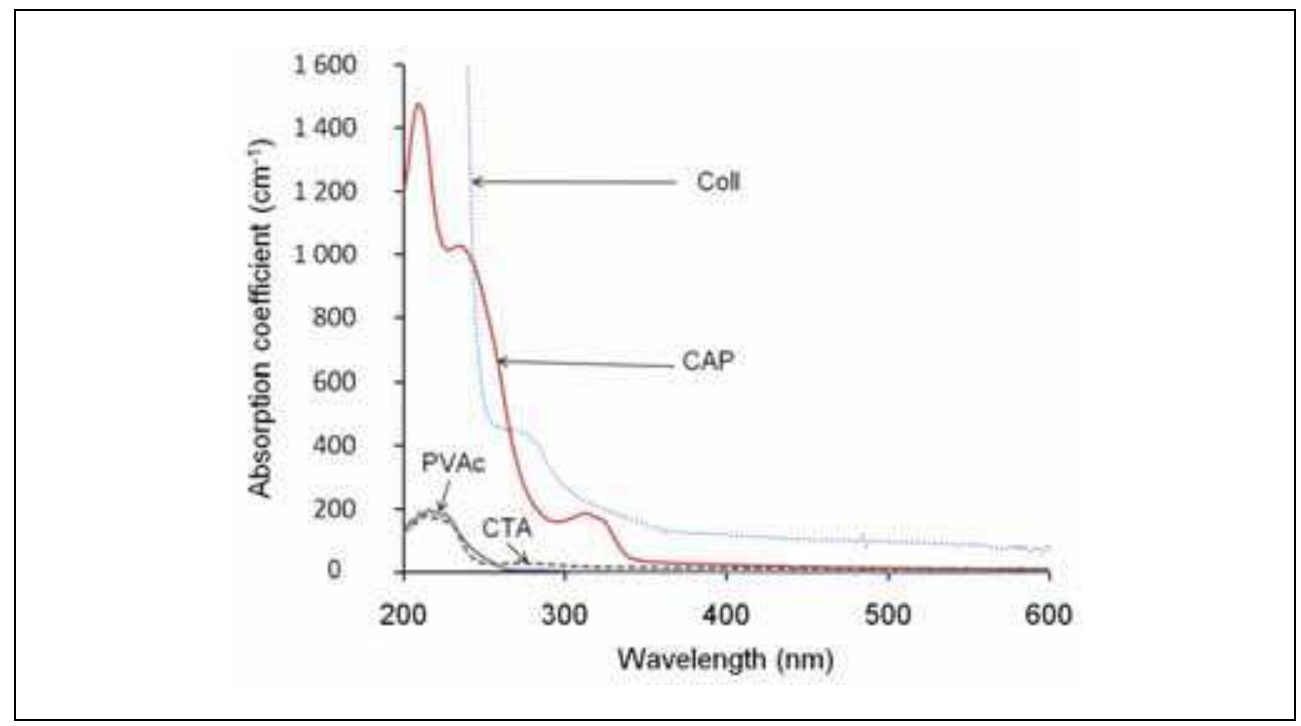

Fig. 4. Absorption spectra of polymer films. The absorption coefficients at the $\mathrm{KrF}$ laser wavelength $248 \mathrm{~nm}$ are (in $\mathrm{cm}^{-1}$ ): CAP/1000; CTA/50; Chi/325; Coll/600; Gel/600.

Absorption of the $\mathrm{KrF}$ laser light at $248 \mathrm{~nm}$ (Fig.4) in the considered polymers can be due to molecular valence transitions of electrons located in chromophores like unsaturations $\left(\pi^{*} \leftarrow \pi\right.$ in $\mathrm{C}=\mathrm{C}$ double bonds) or free doublets located on the oxygen atoms $\left(\pi^{*} \leftarrow n_{\mathrm{O}}\right.$ and $\sigma^{*} \leftarrow n_{\mathrm{O}}$ in $\mathrm{C}=\mathrm{O}$ carbonyl group) or can be due to Rydberg series in which the excited electron has a more diffuse final orbital. Aromatic systems generally give high absorption and are in high concentration in synthetic polymers which display high absorption coefficients of the order of $10^{5} \mathrm{~cm}^{-1}$. On the contrary these unstaturations and aromatic systems are in small concentration in natural polymers, even absent in cellulose derivatives except in CAP in which phthalate groups have been introduced, in low concentration however. In proteins, like collagen, residual aromatic amino-acids include tyrosine, phenylalanine and tryptophan and occurrence is approximately $\sim 1 \%$ of the residues. So, as expected from this consideration, absorption coefficient of collagen $\left(600 \mathrm{~cm}^{-1}\right.$ in fact $)$ is two orders of magnitude smaller than that of a synthetic aromatic polymer $\left(10^{5} \mathrm{~cm}^{-1}\right.$ at $\left.248 \mathrm{~nm}\right)$. In cellulose derivatives non-aromatic esters like acetate yield a rather small absorption which is of the order of $50 \mathrm{~cm}^{-1}$ enough for the foaming experiments with however much larger fluence thresholds compared to higher coefficients. As a consequence the absorption depth $l_{\mathrm{a}}=\alpha^{-1}$ is large and becomes larger than the pressure transit distance during the laser pulse duration. This results in the accumulation of pressure in the absorption depth which makes possible the appearance of the dense cavitation effect. 


\subsection{Experimental results}

In Fig.5 are displayed a few examples of laser-induced nanofoams observed at various magnifications with either the optical or electron microscope. They are all formed with only one laser pulse whose energy density is above the threshold of foaming of the considered polymer. The thickness of the foam layer depends on the target polymer and is of the order of a few tens of microns. It is clearly visible in Fig.5c where a cross-section on a gelatine film is shown. Obviously there is a relationship between energy density of the laser pulse, the absorption coefficient of the material, the foaming threshold and the thickness of the foam layer. With laser pulse energy close to threshold, the onset of the phenomenon, that is to say the formation of a network of micro-bubbles can be observed. Even it has been measured by fast light transmission measurements that some bubbles or cavities are only transiently formed and live only a few microseconds. It is an indication that the gas formed by laser ablation may play an important role in the pressure equilibration inside the bubbles. High fluences are capable of material expulsion and yield more open cells type foamy structure. On the contrary low fluences near threshold tend to give foams with closed cells and probably do not expulse a significant amount of the surface material.

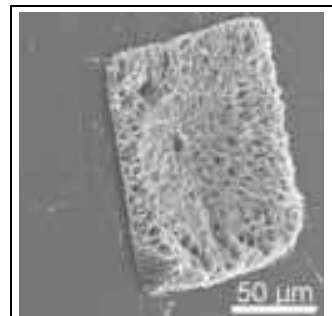

(a)

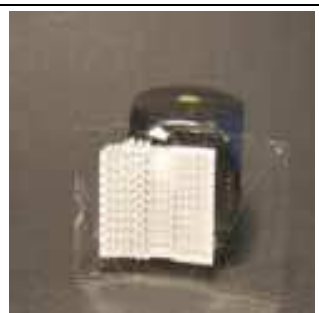

(b)

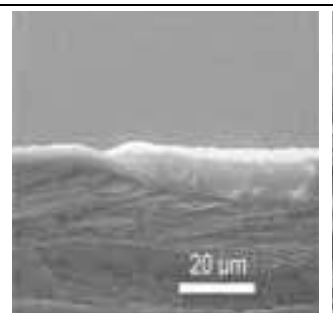

(c)

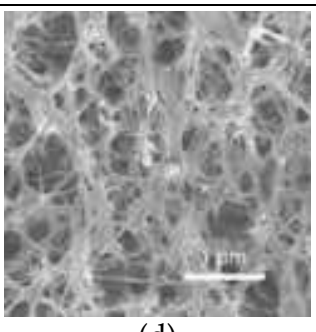

(d)

Fig. 5. Examples of KrF laser induced foams on the surface of (a) collagen film (b) gelatine, macroscopic view (c) gelatine, cross-section (d) chitosan, fine nanofibers network

Poly(vinyl acetate) (PVAc) is studied as a model polymer. Its absorption coefficient at 248 $\mathrm{nm}$ is $30 \mathrm{~cm}^{-1}$ and is well suited to give rise to a strong pressure wave upon absorption of a strong laser pulse. Therefore similar foaming (Fig.6) is obtained although at a higher fluence threshold of $3 \mathrm{~J} / \mathrm{cm}^{2}$. Interestingly PVAc yields the same foaming phenomenon but lends itself more easily to modelling owing to the large body of physical data available in the literature on this synthetic polymer. For all polymers studied so far some general features can be observed like bump formation with a height equivalent to a fraction of the absorption depth, for instance $25 \mu \mathrm{m}$ on PVAc at $6 \mathrm{~J} / \mathrm{cm}^{2}$, a tendency to give closed bubbles near threshold fluence and to give open cell structure at a fluence well above threshold. Moreover the ejected matter which is still poorly known can be solid particles and gases and is probably more abundant at high fluence. Results obtained during experiments with fs laser are reported in details in (Sionkowska et al, 2007; Gaspard et al., 2007, 2008a and 2008b). As shown below they show strong similarity with the ns laser results. Owing to the multi-photon absorption mechanism strongly favoured with fs pulses the radiation wavelength does not need to match the absorption spectrum of the target polymer therefore focusing inside the material makes it possible to create voids in the bulk (Oujja et al., 2009). 


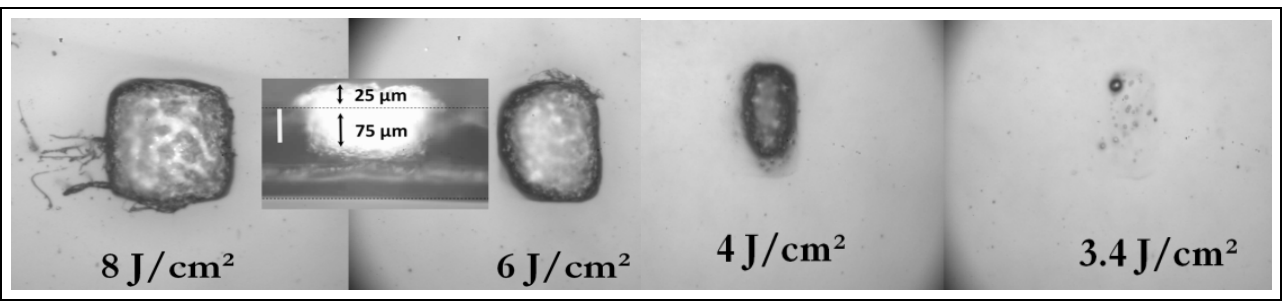

Fig. 6. Ablation spots on a film of poly(vinyl acetate) with one $\mathrm{KrF}$ laser pulse, showing the formation of a microfoam due to the tensile breaking of the polymer. The central inset shows a cross section of the foamed part upon irradiation at $6 \mathrm{~J} / \mathrm{cm}^{2}$ with maximum spot size of $145 \mu \mathrm{m}$.

\section{Fast transmission measurements}

Upon absorption of a laser pulse of energy above threshold, transparent polymer film of this study becomes instantaneously opaque due to the rapid formation of the foamy layer. The rate of appearance of the foam was measured by a specific optical setup (Fig.7) designed to record the data with a time resolution of $1 \mathrm{~ns}$. The light of a probe HeNe laser at $632.8 \mathrm{~nm}$ is normally transmitted without loss before the foam. This transmission of $100 \%$ drops very rapidly within the first $100 \mathrm{~ns}$ after the $\mathrm{KrF}$ ablation laser pulse absorption.

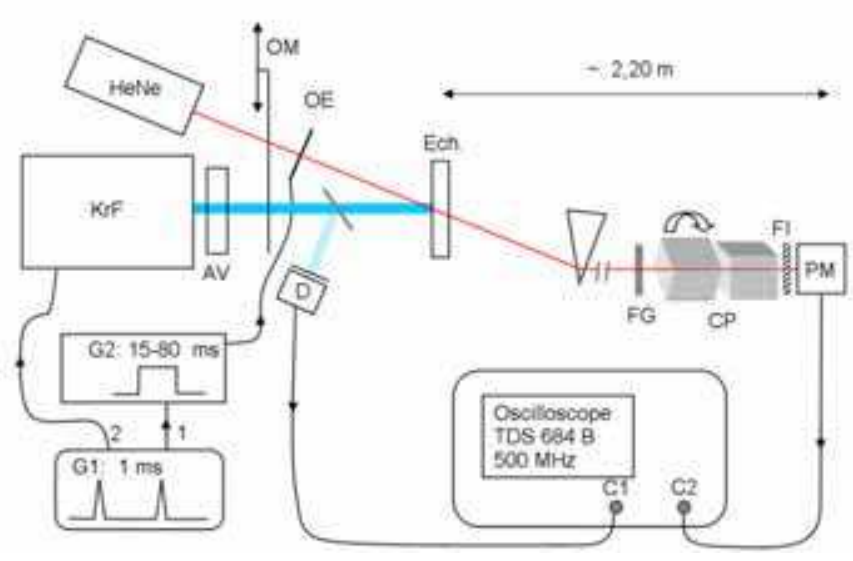

Fig. 7. Setup to measure the fast transmittance drop produced by the laser-induced foam. KrF: Excimer laser 248 nm, HeNe: Laser HeNe 632,8 nm, $5 \mathrm{~mW}$, AV: Variable attenuator, G1: Pulse delay generator (1-5 ms, SRS DG535), G2: Square pulse generator (home made), OE: Electric shutter (Uniblitz, Vincent Associates), OM: Manual shutter, D: Fast Diode Thorlabs DET210 (monitoring of KrF pulse and triggering), Ech. Sample, FG: Grey Filter, FI, Interference filter allowing $632.8 \mathrm{~nm}, \mathbf{C P}$. 2 Polarizing cubes making adjustable attenuation, PM: Photomultiplier 1R446, Oscilloscope: Tektronix digital TDS684 B, 500 MHz, 2,5 Gsamples/s 
To understand this experimental fact one may consider three important data of the present system. The propagation speed of the pressure in the polymer surface of the order of 1 $\mu \mathrm{m} / \mathrm{ns}$, the absorption depth below the polymer surface, $16 \mu \mathrm{m}$ e.g. for collagen and the ablation $\mathrm{KrF}$ laser pulse duration $\sim 30$ ns. Before the more detailed model in sect. 5 and 6 the following simple reasoning can be of valuable help. During the laser pulse absorption and because the volume can be considered as constant the pressure accumulates and does not relaxes before the end of the absorption. In the meantime, the reflexion of the pressure wave on the surface occurs with change of sign, giving rise to a new growing tension wave propagating into the bulk. The cavitation phenomenon does not occur when pressure is high but its nucleation becomes very fast once the compression wave reverses to tension, as will be shown by the model. As a consequence and since tension appears only after a delay time necessary to evacuate the high pressure component of the acoustic wave, the onset of cavitation is observed only $\sim 55 \mathrm{~ns}$ after the beginning of the laser pulse absorption. By using a shorter UV laser pulse with a $90 \mathrm{fs}$ duration, it was shown that this delay is reduced to a lower value of $18 \mathrm{~ns}$ and in full agreement with the model as shown below. The pressure wave dynamics does not depend much on the pulse duration. Changing the duration from $30 \mathrm{~ns}$ to $90 \mathrm{fs}$, that is to say a reduction of $\sim 10^{6}$ only alters the delay time of appearance of cavitation by less of a factor 2 (Lazare at al., 2009).

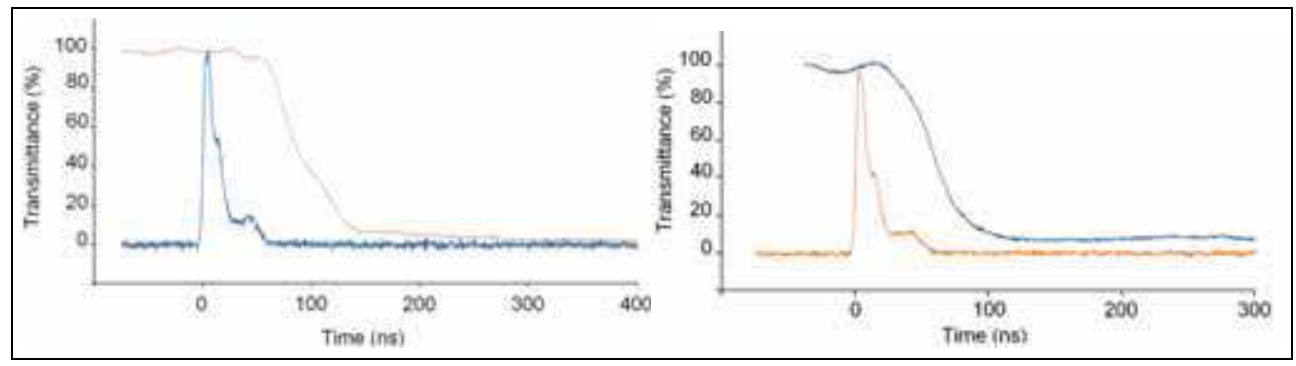

Fig. 8. Example of transmittance fast recording during the $\mathrm{KrF}$ laser-induced foaming phenomenon. Left: for gelatin at $1.7 \mathrm{~J} / \mathrm{cm}^{2}$ and right: for PVAc at $4.5 \mathrm{~J} / \mathrm{cm}^{2}$.

\begin{tabular}{|l|r|r|r|r|r|r|}
\hline Polymer & Abbrev. & $\alpha_{248 n m}\left(\mathrm{~cm}^{-1}\right)$ & $F_{\mathrm{t}}\left(\mathrm{J} / \mathrm{cm}^{2}\right)$ & $\Delta T_{\max }\left(F_{t}\right)$ & $\Delta P\left(1 \mathrm{~J} / \mathrm{cm}^{2}\right)$ & $T_{\mathrm{g}}(\mathrm{K})$ \\
\hline Gelatin & GEL & 600 & 0.5 & 144 & $90 \%$ & 434 \\
\hline Collagen & COL & 600 & 0.5 & 144 & $90 \%$ & \\
\hline Chitosan & CHI & 325 & 1.5 & 260 & $67 \%$ & \\
\hline Cellulose triacetate & CTA & 50 & 4 & 100 & $19 \%$ & 378 \\
\hline Cellulose dinitrate & CDN & 430 & & & & 333 \\
\hline $\begin{array}{l}\text { Cellulose Acetate } \\
\text { Phthalate }\end{array}$ & CAP & 1000 & 0.15 & 74 & $100 \%$ & 348 \\
\hline Poly(vinyl acetate) & PVAc & 30 & 3 & 60 & $10 \%$ & 305 \\
\hline Poly(vinyl pyrrolidone) & PVP & 120 & 1.5 & 90 & $43 \%$ & 448 \\
\hline
\end{tabular}

Table 1. Physical data of the polymers considered for the laser-induced foaming phenomenon. Target temperature is $25^{\circ} \mathrm{C}$ just before laser pulse. 


\section{Theory of laser-induced strong pressure wave}

Three parameters define the state of the matter excited by the laser pulse during and after the absorption step. Temperature, pressure and volume are time dependent as a characteristic of the non-equilibrium due to the short pulse laser excitation and can be estimated by the following theoretical approach. The dynamics of temperature and pressure are very different because heat diffusion is slow in polymers and pressure relaxes by propagation at the speed of sound. Therefore for the time window needed to be considered, the temperature profile is stationary and given by:

$$
T(z, t)=\frac{\alpha A_{0}}{C_{p} \rho} F_{0} e^{-\alpha z}+T_{0}=\Delta T_{\max } e^{-\alpha z}+T_{0}
$$

with the signification of the symbols given in the nomenclature below. This temperature elevation is the origin of the pressure rise and its profile is not expected to vary in the timescale of the foaming transition (100 ns) since the appropriate model shows that $10 \mu \mathrm{s}$ are necessary to display a significant evolution of the profile by diffusion, a time much longer than the foaming appearance. As seen in Table 1 the maximum temperature elevation at threshold, as estimated by the model, is for most polymers very low and is very rapidly averaged and applies for durations of the order of milliseconds. It suggests that temperature alone does not account for the observed transition. Several other factors have to be considered like pressure elevation and ablation products. Ablation products are usually very diverse since they can be gases, polymers, particles and resulting of thermal or photochemical reactions. To consider them is important because they are the driving force that makes the foaming process possible once the tension wave comes into play. The pressure is considered now as the result of the temperature elevation before the volume expansion that occurs at finite speed, the speed of sound $c_{\mathrm{s}}$. As soon as volume expansion is possible and occurs, pressure decreases. The dynamics of the pressure wave is governed by its propagation equation:

$$
\frac{\partial^{2} P(z, t)}{\partial^{2} t}-c_{s}^{2} \nabla^{2} P(z, t)=-\frac{\beta}{C_{P}} \frac{\partial[A \alpha F(z, t)]}{\partial t}
$$

A one dimension model is assumed since the spot size of diameter larger than $500 \mu \mathrm{m}$ implies a diffusion along the z-axis. This type of equation does not take into account the zattenuation of the pressure wave due to bulk and shear viscosity because only the short distance propagation is considered. One is interested in the pressure developed within the absorption length and not more. This approximation has to be reconsidered for a larger propagation. The solution to the propagation equation (2) can be easily described for an ultrashort (e.g. $\tau=90 \mathrm{fs}$ ) pulse of incident fluence $f_{0}$ for which the confinement is total, that is to say no pressure relaxation at the end of the pulse. In that case almost all the absorbed energy contributes proportionally to the pressure elevation according to the Mie-Grüneisen equation of state of the target material (Lazare et al., 2009).

$$
\begin{gathered}
\Delta p(z, t=\tau)=\Gamma \cdot \alpha \cdot f_{0} \cdot e^{-\alpha \cdot z}=p_{\max } \cdot e^{-\alpha \cdot z} \\
\Gamma=-\left(\frac{\partial \ln P}{\partial \ln (U / V)}\right)_{V}=\frac{c_{s}^{2} \beta}{c_{p}}
\end{gathered}
$$




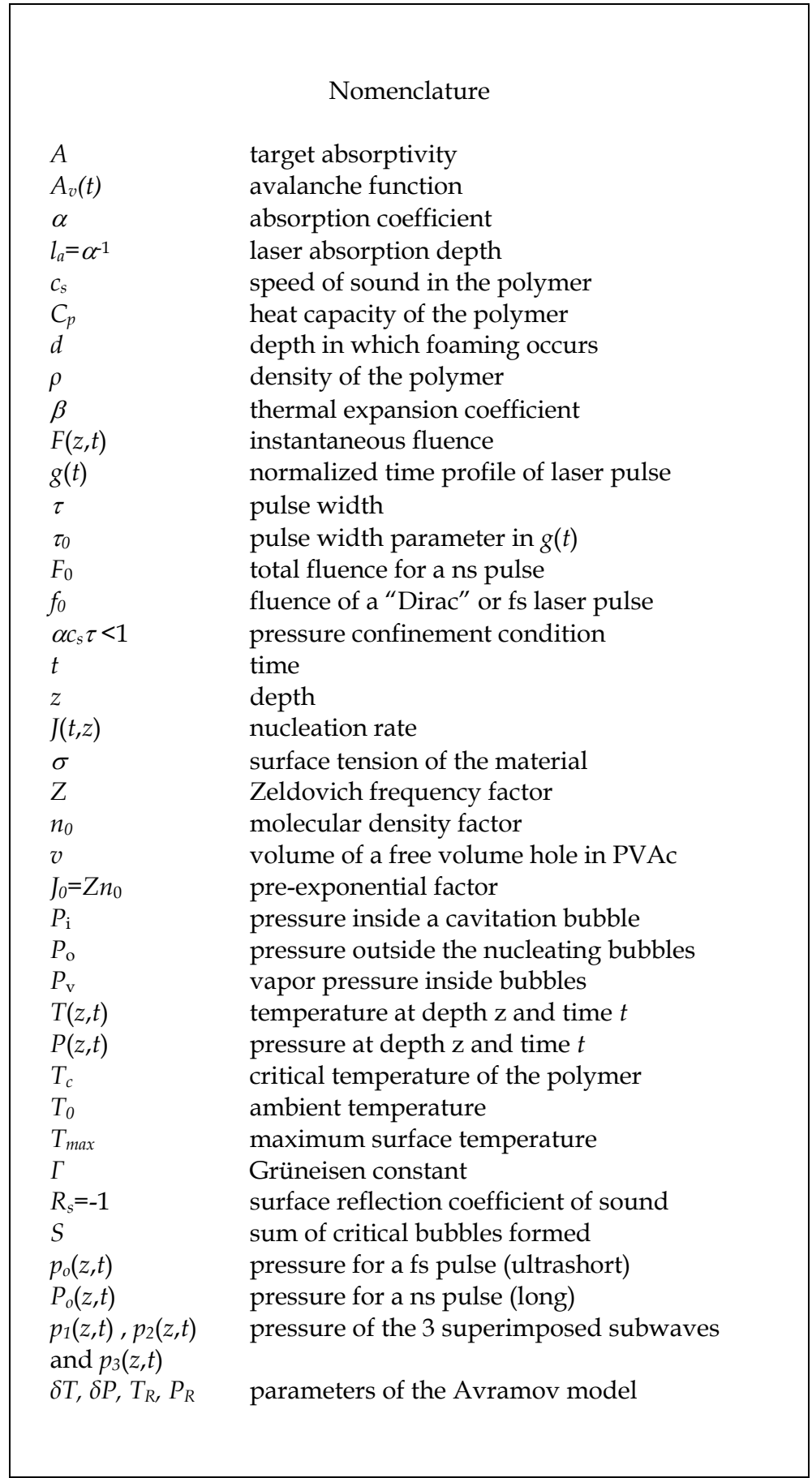


The proportionality constant $\Gamma$ is called the Grüneisen coefficient and is approximated to 1 in the case of polymers.

In fact the considered pressure elevations are of the order of $\sim 10-10^{5}$ bar, high values compared to ambient pressure which can be neglected in the formulation. The physical solution to (2) can be explicited as a sum of three sub-waves $p_{1}, p_{2}, p_{3}$, with one $p_{2}$ being reflected on the surface of the sample to yield $p_{3}$ with a reflection coefficient $R_{s}=-1$.

$$
\begin{gathered}
p_{0}(z, t)=\Delta p(z, t)=p_{1}(z, t)+p_{2}(z, t)+p_{3}(z, t) \\
p_{1}(z, t)=0.5 p_{\max } e^{-\alpha\left(z-c_{s} t\right)}, \text { if } z>c_{s} t, \text { otherwise } 0 \\
p_{2}(z, t)=0.5 p_{\max } e^{-\alpha\left(z+c_{s} t\right)}, \text { if } z>0, \text { otherwise } 0 \\
p_{3}(z, t)=0.5 p_{\max } R_{s} e^{-\alpha\left(c_{s} t-z\right)}, \text { if } c_{s} t>z>0, \text { otherwise } 0
\end{gathered}
$$

This sub-wave number 3 has a negative sign due to $R_{\mathrm{s}}=-1$ and is associated with the tension wave that can be observed in the experiments. The absorption of the laser ultrashort pulse launches two counter-propagating compression sub-waves and one is reflected on the surface with the formation of a retro-propagating tension wave. As the surface compression is converted into tension with momentum conservation, in the theoretical absence of energy dissipation due to viscosity. It appears progressively and after relaxation of the compression sub-waves. The tension wave develops enough intensity to induce cavitation after some propagation time which can be evaluated in the case of an ultra-short pulse by:

$$
\Delta t \cong \frac{1.5}{\alpha c_{s}}
$$

The applicability of this consideration has proved to be successfull by comparing the ultrashort laser pulse (90 fs at $266 \mathrm{~nm}$ ) case with the $\mathrm{ns} \operatorname{KrF}(25 \mathrm{~ns}$ at $248 \mathrm{~nm}$ ) laser pulse case. The absorption depths are assumed to be close if the non-linear absorption of the ultra-short pulse is negligible (it is the case at low fluence). Now the pressure wave $P_{0}(z, t)$ due to the absorption of a long $\mathrm{KrF}$ pulse is evaluated as the result of the absorption of discrete series of many ultra-short laser pulses equally spaced and with allowance for relaxation between two elementary pulses. It is therefore the convolution product which is developed below. Let us consider a theoretical $\mathrm{KrF}$ laser pulse time profile of the following form:

$$
F(t)=F_{0} \int_{0}^{t} g\left(t^{\prime}\right) d t^{\prime}=F_{0}\left(\int_{0}^{\infty} t^{2} e^{-t / \tau} d t\right)^{-1} \int_{0}^{t} t^{2} e^{-t^{\prime} / \tau} d t^{\prime}
$$

It is normalized to be equal to the incident fluence at the end of the pulse and its duration is adjusted to the experimental one with the parameter $\tau=7.5 \mathrm{~ns}$. The function $\mathrm{g}(\mathrm{t})$ is the time envelop or profile of the $\mathrm{KrF}$ laser pulse and of the successive theoretical elementary ultrashort pulses. If one considers an isolated "Dirac delta pulse" delivered at time $t_{1}$, at time $t_{2}$ after relaxation during $t_{2}-t_{1}$ the pressure elevation due to this pulse is $p_{0}\left(z, t_{2}-t_{1}\right)$. The sum of all pressure elevations is the given by the convolution product:

$$
P_{0}(z, t)=p(z, t) \otimes g(z, t)=\int_{0}^{t} g\left(t_{1}\right) p_{0}\left(t-t_{1}\right) d t_{1}
$$


This model of pressure wave accounts well for the confinement effect which occurs when the absorption depth $l_{a}=\alpha^{-1}$ increases up to an optimum above which it decreases again at constant fluence because the absorbed energy density becomes too low. Two competing factors are therefore to be considered in the experiment, one varies like $\alpha$ and the other like $\mathrm{e}^{-\alpha}$. This sensitivity of polymers to develop pressure confinement is expressed in the Table 1 by $\Delta P$ the pressure increase as a function of $\alpha$ for an incident pulse of $1 \mathrm{~J} / \mathrm{cm}^{2}$. It is predicted that the best value of absorption coefficient giving the most intense acoustic pressure is for $\alpha=1000 \mathrm{~cm}^{-1}$ which is that of CAP, for instance. Examples of pressure waves are displayed in Fig.9 for two different polymers with significantly different absorption coefficients. It is seen that PVAc develops a stronger acoustic wave because the fluence needed for the ablative foaming is one order of magnitude larger.

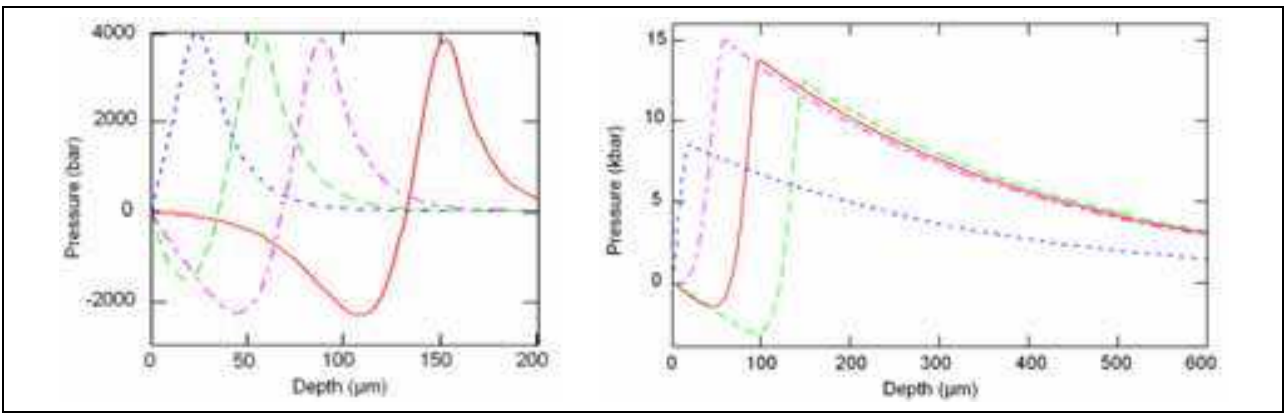

Fig. 9. Examples pressure depth profiles of acoustic waves propagation for two significantly different polymers: $\underline{\text { left }}$ is collagen or gelatine with same $\alpha=600 \mathrm{~cm}^{-1}$ at $t=20,40,60$ and 100 ns, fluence is $F_{0}=0.5 \mathrm{~J} / \mathrm{cm}^{2}$; $\underline{\text { right }}$ is PVAc with lower $\alpha=30 \mathrm{~cm}^{-1}$ at $t=20,60,100$ and $150 \mathrm{~ns}$ for $F_{O}=6 \mathrm{~J} / \mathrm{cm}^{2}$.

The important feature of the acoustic profile is the negative component that develops with time after some propagation in depth. It corresponds to the tensile state of the material and its increasing intensity with depth is at some critical point capable of creating the cavitation phenomenon which is the topic of this work. In the approximations of this model the parameter $c_{\mathrm{S}}$ can be adjusted by fitting the experimental data of Fig.8 so that the appearance time of the foam is predicted by the cavitation kinetic theory. However since the data do not allow a high precision $c_{\mathrm{s}}$ is normalized to the realistic value of $1 \mu \mathrm{m} / \mathrm{ns}$ in the following. Further comments can be made on the transition fall from high compression peak to the tensile component maximum. It is called the rarefaction wave since a fast density decrease is associated to it. In the absence of absorption it is propagating into the bulk, like the acoustic pressure wave without change in profile. Its width or steepness is characteristic of the laser pulse duration which can be varied dramatically by changing the laser type and pulse duration. Taking an ultrafast laser pulse with a 90 fs duration makes it very narrow compared to the $\mathrm{KrF}$ ns laser which has a $10^{6}$ time larger pulse width.

\section{Kinetic theory of dense cavitation}

The foaming process develops in the absorption zone because of the slight temperature elevation and is triggered by the acoustic wave. Its kinetics distributed in space and time $J(z, t)$ can be expressed by the classical theory of nucleation (Zeldovich, 1942). 


$$
J(z, t)=J_{0}(z, t) e^{\frac{\Delta G(z, t)}{k T(z, t)}}=n_{0} \frac{\sigma(z, t)}{\eta(z, t)} \sqrt{\frac{\sigma(z, t)}{k T(z, t)}} \exp \left(-\frac{16 \pi \sigma(z, t)^{3}}{3 k T(z, t)\left[P_{i}(z, t)-P_{0}(z, t)\right]^{2}}\right)
$$

This rate expression in (8) has a classical exponential factor with the free-energy barrier $\Delta G$ to form the critical bubble depending on liquid surface tension and the inner and outer pressure difference $P_{\mathrm{i}}-P_{\mathrm{o}}$. The pre-exponential factors are $n_{0}$ a molecular concentration and the Zeldovich frequency factor $Z$ which includes surface tension and viscosity of the liquid in this case. Although various forms of $Z$ have been introduced by several authors, this particular one taken in (Baidakov, 2007) has been chosen because it shows an interesting dependence on viscosity. Almost all material related quantities like viscosity and surface tension are temperature and pressure dependent, consequently time and depth dependent, a difficulty which imposes large approximations. The evaluation of the molecular concentration $n_{0}$, called sometime number density, is a real problem in the present case of voids concentration contrary to the usual kinetic theory in an elemental liquid like the rare gas liquids. However since the rate $J$ quantifies bubbles or cavities formed upon the stretching situation created by the laser pulse absorption one can look for the concentration of their possible precursors in the starting polymer. The possible candidates for yielding bigger bubbles after critical expansion are the free volume holes that are well known and present in the condensed matter in general. In molecular liquids their presence and concentration is tightly related to the concentration and shape of the molecule. In polymers the free volume holes distribution reflects the complexity of the chain molecules. Below we discuss the case of PVAc for which extensive literature data is available and which provides numerical data of rate. In a previous work (Lazare et al. 2009) the ratio $J / J_{0}$ was used to explore the dynamics capable of interpreting the transmission curves of Fig.8. By doing so, only the exponential factor is considered in the evaluation of the avalanche function $A_{\mathrm{v}}(t)$ which was used to compare long ns pulse to ultrashort fs foaming of Fig.10.

$$
A_{v}(t)=100\left(1-S(t) / S_{\infty}\right)
$$

$S(t)$ is the sum of the critical bubbles formed at time $t$ in the considered depth and $S_{\infty}$ at $t=\infty$.

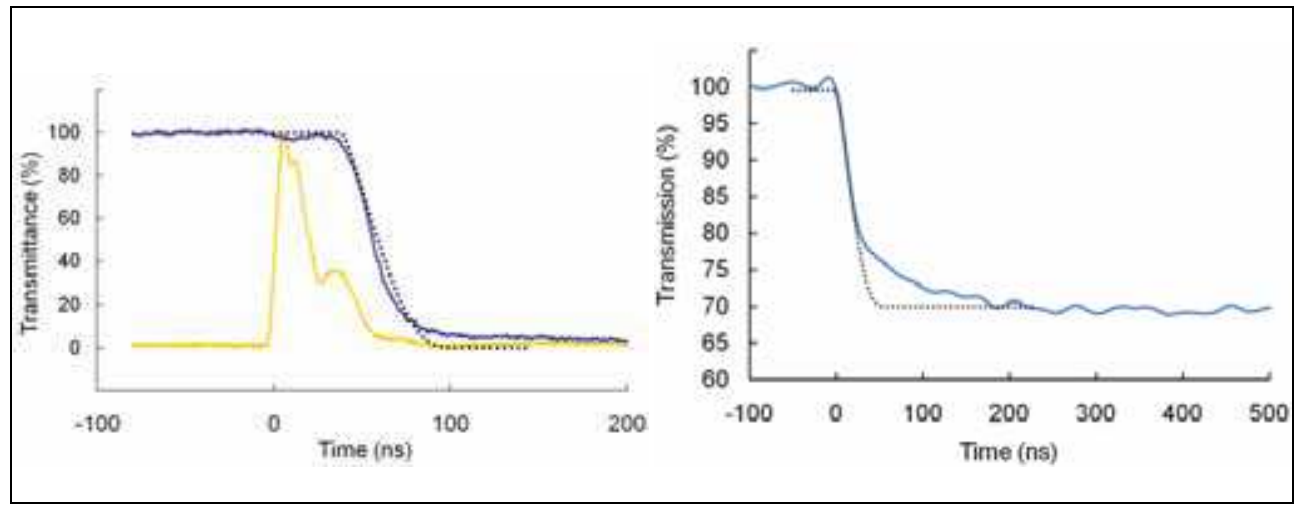

Fig. 10. Transmittances and predicted theoretical avalanche function $A_{\mathrm{v}}(t)$ for a $\mathrm{KrF}$ ns pulse (left) and an ultrashort fs pulse (right). 
The accumulated bubble since the beginning of the laser pulse absorption is given by:

$$
S(t)=\int_{0}^{t} \int_{0}^{d} J\left(t^{\prime}, z, F_{0}\right) d z d t^{\prime}
$$

In which $J$ is explicitly given by a combination of (1), (7) and (8). Necessarily the depth of integration $d$ is limited to a realistic value usually taken slightly larger than $l_{\mathrm{a}}$, for example $d=20 \mu \mathrm{m}$ for collagen having $l_{\mathrm{a}}=16 \mu \mathrm{m}$.

\begin{tabular}{|c|l|l|l|l|}
\hline Collagen & $\rho=1.3 \mathrm{~g} / \mathrm{cm}^{3}$ & $\sigma=30 \mathrm{~mJ} / \mathrm{m}^{2}$ & $C_{\mathrm{p}}=1.5 \mathrm{~J} / \mathrm{g} . \mathrm{K}$ & $C_{\mathrm{s}}=1 \mu \mathrm{m} / \mathrm{ns}$ \\
\hline
\end{tabular}

Table 2. Data used for the collagen model

The model predicts a sigmoid curve that can be fitted to the experimental data as in Fig.10 if the speed of sound is $c_{\mathrm{s}}=1 \mu \mathrm{m}$. The other major approximation brought to the model concerns the pressure of ablation gas $P_{\mathrm{i}}$ that definitely contributes to the increase of the bubble growth. However it can be neglected in some part of the time window without significant loss of prediction power, but may play a role in the mechanism depending on the experimental parameters. In these conditions, if it is assumed that the tensile state alone is enough to reach a critical state that triggers the intense foaming, so a good prediction of the experimental data is obtained like in Fig.10 for both ns and fs regimes. It is observed that foaming does not occur in the high pressure compressive first component of the acoustic wave but during the rarefaction component, that is to say in the tensile tail. The nucleation wave propagation coincides with that of the tensile component as shown by the model for collagen (Fig.11). Furthermore the experimental value of the threshold fluence is well predicted by the present model. The main governing data of the threshold prediction are in table 2 and can be refined if a better fitting is needed. Among them probably the most determinant one is the surface tension $\sigma$. The lack of reliable data on viscosity of the biopolymer in order to be able to fully explicit the pre-exponential factor in (8) led us to consider the case of a new model polymer PVAc (see next section). In section 7 it is shown that the introduction of a model of viscosity with $T$ and $P$ dependence opens a full access to the numerical values of the voids nucleation rates

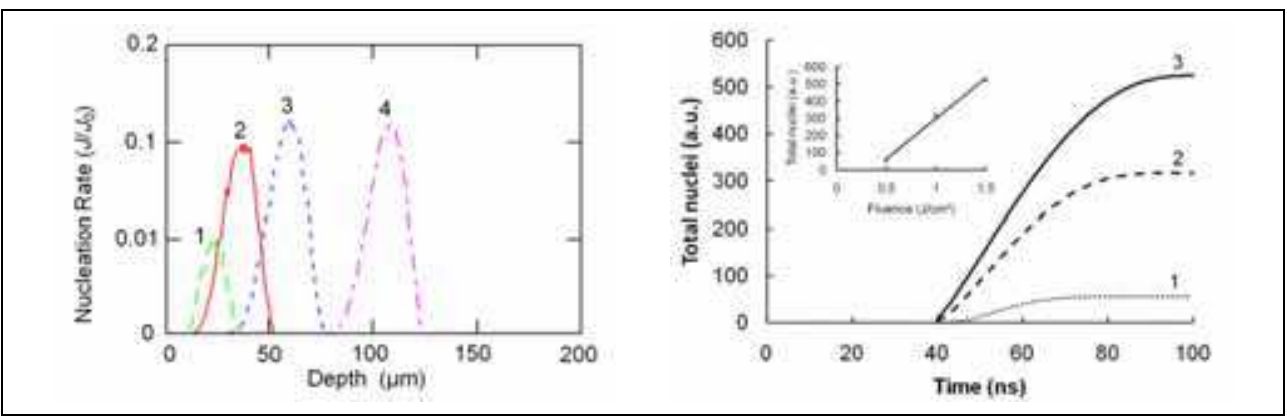

Fig. 11. (left) Nucleation wave in collagen for a $\mathrm{KrF}$ laser pulse of $F_{0}=0.5 \mathrm{~J} / \mathrm{cm}^{2}$ for various time (1) $\mathrm{t}=45$ (2) 55 (3) 70 (4) $100 \mathrm{~ns}$. (right) Total number of critical bubbles as a function of time at various flunces (1) $F_{0}=0.5$ (2) 1 (3) $1.5 \mathrm{~J} / \mathrm{cm}^{2}$. The inset shows the ablation curve predicting the experimental value. 


\section{Case of a model polymer (PVAc)}

The data on laser-induced foaming of PVAc are presented in Fig.6, 12 and table 1. Further details are given in (Lazare et al., 2010). The case of this synthetic polymer simplifies the modeling owing to its molecular structure made of a simpler repeat unit. Therefore it has lent itself to many theoretical and physical studies, concerning viscosity of interest in this work and specially the temperature and pressure dependence.

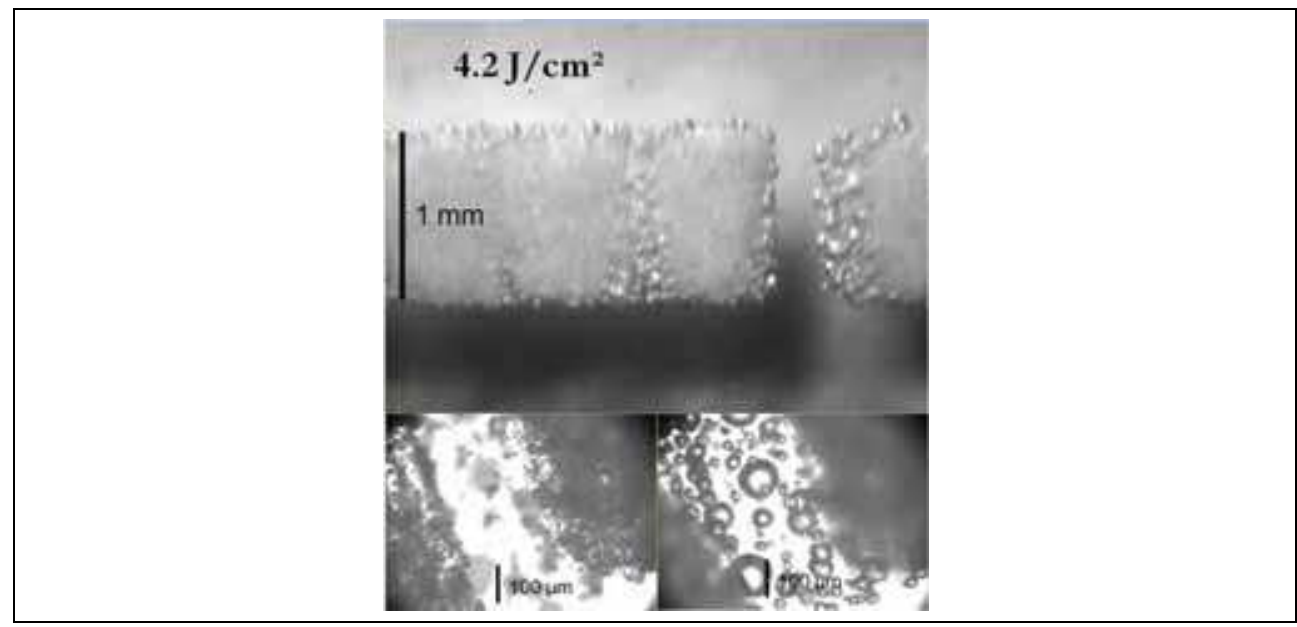

Fig. 12. Poly(vinyl acetate) PVAc film foaming with single pulses of the $\mathrm{KrF}$ laser at $4 \mathrm{~J} / \mathrm{cm}^{2}$. Several spots show small bubbles on surface and larger bubbles deeper in the film.

Examples of large KrF laser spots are shown in Fig.12 main picture which reveals some characteristic features like bubbles bigger than in the case of biopolymers. This may be explained by the larger absorption depth and the lower glass transition temperature $T_{\mathrm{g}}$ which allows to reach lower viscosity and higher bubble diffusion at lower temperature. In the bottom two-pictures which focus on the same spot but at two different depths in the polymer, it can be seen that the surface bubbles are smaller and conversely larger more in depth (right picture). The model predicts that the tensile component becomes larger with propagation in depth, therefore the viscosity does the same and since the diffusion varies like the reciprocal of the viscosity, it is thought that bubble collisions and growths are increased. Polymer melt viscosity is an important topic for the applications like thermal processing and molding. Therefore the literature is wide and reports many models of temperature-"only" dependence like Williams-Landel-Ferry (WLF) (Williams et al., 1955) and the (Angell, 1985) (AM). Although the $P$ dependence alone is discussed for a long time since (Barus, 1891), the development of simultaneous $T$ and $P$ dependent viscosity models $\eta(T, P)$ is more recent and can be provided by (Avramov \& Milchev, 1988), later adapted to PVAc by (Roland \& Casalini, 2003) in equation (11) where $\delta_{T}, \delta_{P}, T_{R}, P_{R}$ are parameters characteristic of the material.

$$
\eta(T, P)=\exp \left[30\left(\frac{T_{R}}{T}\right)^{\delta T}\left(1+\frac{P}{P_{R}}\right)^{\delta P}\right]
$$




\begin{tabular}{|c|c|c|c|c|}
\hline$\rho=1.19 \mathrm{~g} / \mathrm{cm}^{3}$ & $C_{\mathrm{p}}=1.5 \mathrm{~J} / \mathrm{g}$ & $\sigma_{0}=36.5 \mathrm{~mJ} / \mathrm{m}^{2}$ & $c_{\mathrm{s}}=1 \mu \mathrm{m} / \mathrm{s}$ & $n_{0}=0.77 \times 10^{21} \mathrm{~cm}^{-3}$ \\
\hline$\delta_{T}=7.11$ & $\delta_{P}=1.62$ & $T_{R}=302.2 \mathrm{~K}$ & $P_{R}=2710 \mathrm{bar}$ & $T_{c}=1000 \mathrm{~K}$ \\
\hline
\end{tabular}

Table 3. PVAc physical data used in the modelling

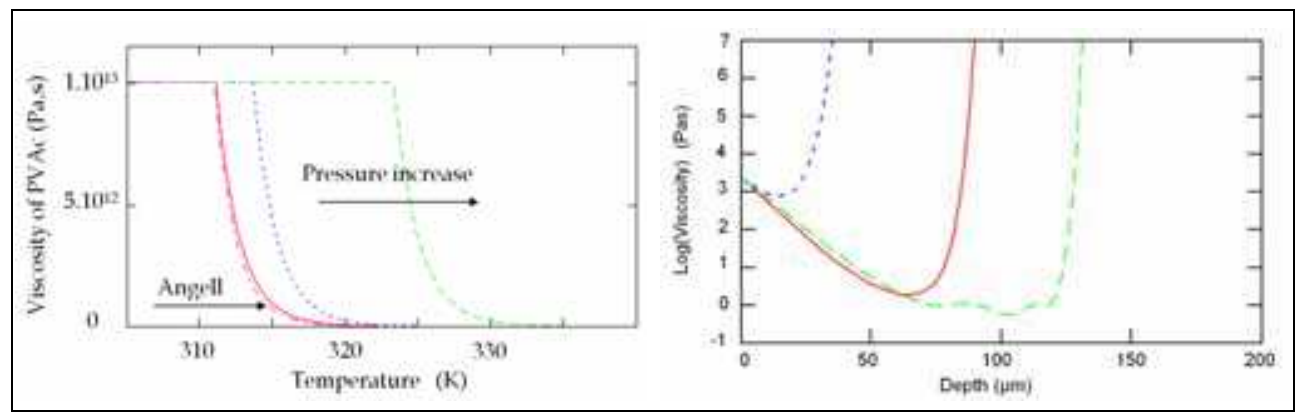

Fig. 13. (left) Poly(vinyl acetate) PVAc viscosity dependence on temperature and pressure (1, 10 and 50 bar) with the Avramov model equation (11) and comparison with Angell model at 1 bar. (right) $\mathrm{KrF}$ one pulse $\left(F_{0}=6 \mathrm{~J} / \mathrm{cm}^{2}\right)$ laser-induced viscosity change at various times 70 , 120,160 ns.

The numerical model of viscosity with temperature and pressure dependence is presented in Fig.13 with the physical data of PVAc shown in table 3. As seen an increase of pressure results in an increased viscosity so that during the laser pulse absorption the $\Delta T, \Delta P$ couple can be so that the absorbing layer remains solid up to the rarefaction wave time. This explains why in the transmission recording experiments of Fig.8 and 10, the blackening reflecting the appearance of the avalanche of bubbles occurs well after the end of the laser pulse. As suggested in the simulation Fig.13 (right) of the time evolution of the depth viscosity profile in the PVAc film the polymer of low viscosity appears only at $t \sim 100 \mathrm{~ns}$ and in a layer located at $z>25 \mu \mathrm{m}$ below the surface. In the same line the rate of nucleation must be in agreement with this picture. The model of surface tension of the polymer is provided by the (Guggenheim, 1945) equation (12) which is only temperature dependent since the pressure dependence is not known.

$$
\sigma(T)=\sigma_{0}\left(1-T / T_{c}\right)^{11 / 9}
$$

Before going further it important to give some background next section on the considerations on the Free Volume Theory, because it provides some numerical value of $n_{0}$, the concentration of free volume holes, in equation (8) and also it plays a role in the variation of viscosity with temperature and pressure.

\subsection{Free volume theory}

The free volume existence is common to any condense phase. For a long time, its two components, the interstitial free volume and the free volume holes are known and studied. Both types can be found in polymers (Budd et al., 2005) like any solid or liquids, however 
free volume holes are nanometer size and more frequent in polymer phases for statistical reasons due to the macromolecular character of the molecule. It is well known that at the solid to liquid transition of polymers the hole free volume fraction increases faster with temperature than the molecular volume and the interstitial free volume (Fig.14). This means that either their number, or their size, or both increase by thermal expansion and confers new properties to the polymer like decrease of viscosity, increase of diffusion and permeability, etc.. The interstitial free volume is the unoccupied space between atoms, which is necessarily present even at $0 \mathrm{~K}$ and increases with temperature like the molecular volume expansion.

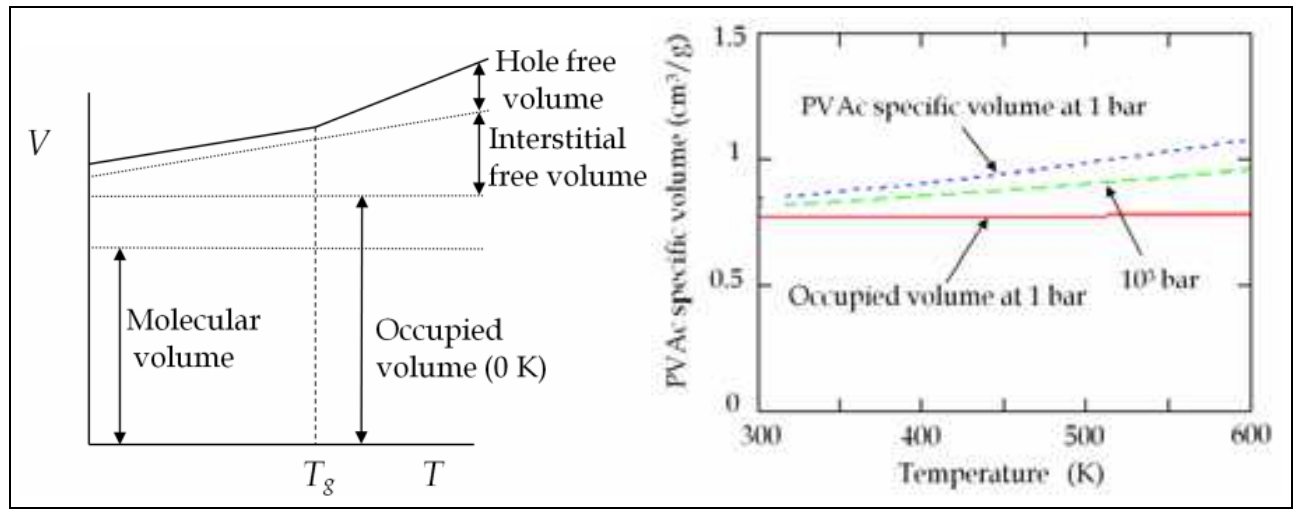

Fig. 14. (left) Temperature variation of the specific volume of polymers around Tg and its contributive components. (right) PVAc theoretical specific volumes variations with temperature at 1 and $10^{3}$ bar calculated with the Simha equation of state (Dlubek et al., 2000). The difference between occupied and specific volumes at 1 bar is due to the free volume holes.

Holes of free volume (fvh) can be reduced in size and number by decreasing temperature and by processes like physical aging. Their concentration and volume are a fraction of that of the constitutive molecules and are known experimentally from dilatometry (McKinney \& Belcher, 1963), X-ray diffraction (Wendorf \& Fischer, 1973), ortho-positronium anhilation lifetime spectroscopy (o-PALS) (Tao, 1971; Dlubek et al., 2000; Hong-ling et al., 2008). This experimental knowledge is predicted by the theoretical equation of state (SS-EOS) developed by (Simha \& Somcynski, 1969) using the cellular method of (Lennard-Jones \& Devonshire, 1937). In the work of (Dlubek et al., 2000) a numerical solution of the theoretical SS-EOS can be found and used to compute the specific and hole free volumes of PVAc in Fig.14 (right). The plot shows the importance of the hole free volume that increases with temperature, whereas the occupied volume increases only slightly. At higher applied pressure 1000 bar, a normal compression phenomenon occurs. These data are for an equilibrium situation only and have to be used with caution when interpreting the laser experiment since the timescale, the dynamic windows and the fast evolution are placing the system in a region out of equilibrium. Nevertheless SS-EOS data are useful to characterise the starting polymer system and its free volume holes. The precise o-PALS results provide the concentration free volume holes $\left(n_{0}=0.77 \times 10^{21} \mathrm{~cm}^{-3}\right)$ in PVAc and the mean hole volume $\left(0.1 \mathrm{~nm}^{3}\right)$ at room temperature. This provides a satisfactory value of $n_{0}$ in our calculation of 
nucleation rate (8) for PVAc (see next section). Furthermore it appears very likely that at the rarefaction wave the ablation gas can diffuse to the inner of these fvh. Just before rarefaction the high pressure maintains the system in a solid state with high viscosity and one may consider that ablation gas are blocked in the polymer or are not yet formed. For PVAc ablation gas products obtained with a $\mathrm{CO}_{2}$ laser have been reported to be mainly $\mathrm{H}_{2}, \mathrm{CO}$, $\mathrm{CO}_{2}, \mathrm{CH}_{3} \mathrm{COOH}, \mathrm{CH}_{3} \mathrm{COOCHCH}_{2}$ (Kupcik et al., 2005). Most of these molecules have sizes that are small enough to be contained in the original fvh, however at rarefaction an expansion of the system increases dramatically the diameter of the holes and amplifies the diffusion phenomenon which builds up the inner gas pressure in the nucleating bubbles.

\subsection{Stretched state of the polymer}

Evaluating the nucleation rate (8) requires some approximation to be considered as far as ablation gas pressure $P_{\mathrm{i}}$ inside the growing bubbles is concerned. As in our previous work one may consider that $P_{\mathrm{i}}$ can be neglected and that the tensile state of the polymer is enough to create the new voids. As shown this yields a polymer in a stretched state which is well capable of high nucleation rate in the condition of the experiments. The case of PVAc is shown in Fig.15 and reveals the dependence of the nucleation rate on time and fluence. The onset of nucleation occurs at a well defined depth of $27 \mu \mathrm{m}$ and starts at a time of $\sim 60 \mathrm{~ns}$. The calculation further shows that pre- and exponential factors in (8) grow simultaneously with time but with different rates. In the present case of PVAc the pre-exponential factor is the limiting one. This seems to indicate that viscosity is the controlling parameter of the system when $T$ and $P$ vary in a large range during the pulsed heating. The inset of Fig.15 indicates that above the predicted threshold which fits well the experimental one of $3 \mathrm{~J} / \mathrm{cm}^{2}$ (table 1), there is a strong accumulation of the post-nucleation bubbles.

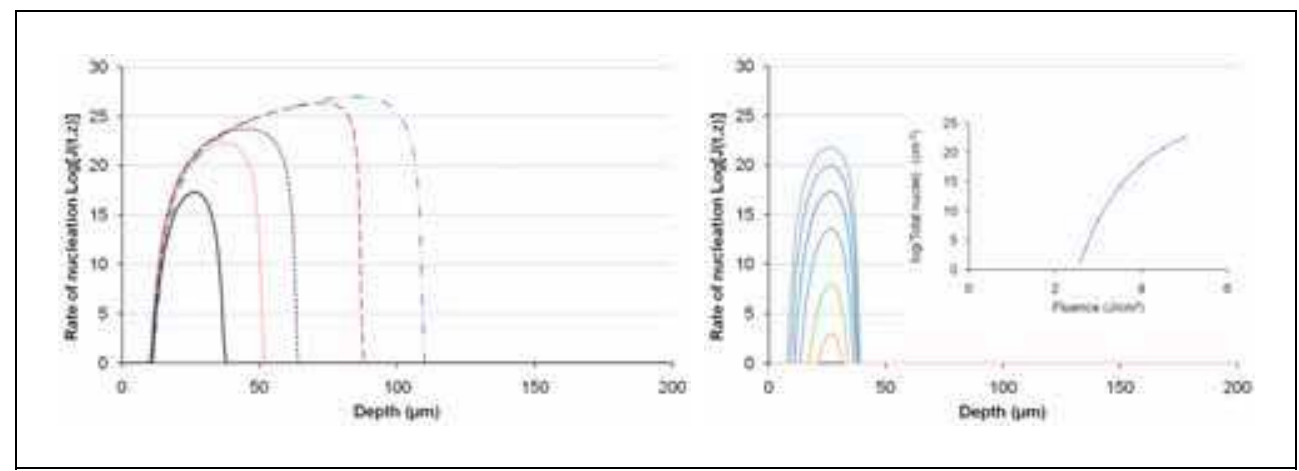

Fig. 15. (left) Theoretical rate of nucleation (in nucl. $\mathrm{cm}^{-3} \mathrm{~s}^{-1}$ ) as a function of depth in PVAc at various times $80,90,100,120,140 \mathrm{~ns}$ after the laser pulse absorption $\left(F_{0}=4 \mathrm{~J} / \mathrm{cm}^{2}\right)$. (right) Theoretical rate of nucleation (in nucl.cm $\left.{ }^{-3} \mathrm{~s}^{-1}\right)$ as a function of fluence $\left(F_{0}=2.7,3.0,3.5,4.0\right.$, $4.5,5 \mathrm{~J} / \mathrm{cm}^{2}$ ) in PVAc at a time of $80 \mathrm{~ns}$ after the pulse absorption. The inset is the total number of nuclei formed at $80 \mathrm{~ns}$ as a function of fluence.

These results also show that cavitation does not occur selectively at the extreme surface of the polymer as would be suggested by the laser temperature or excitation profile but rather at the depth where the tension wave reaches a maximum intensity. Moreover cavities, free volume holes and nanobubbles formed in the stretching phase of the mechanism are 
expected to facilitate the molecular diffusion according to the principle of (Cohen \& Turnbull, 1959). As expressed with the Stoke-Einstein equation (13) the diffusion coefficient $D$ varies like the reciprocal viscosity in a system with spherical molecules of radius $r$.

$$
D=\frac{k T}{6 \pi \eta r}
$$

It follows that nano-bubbles collisions and merging is activated by the "in-depth" viscosity drop giving rise to the birth of larger bubbles like that seen in Fig.12 deeper below the surface. Nano-cavities merging is also a mechanism which can help the system reach the critical state faster at some points. Now if some ablation gas pressure model $P_{\mathrm{g}}(z, t)$ is added to the present system, then equation (8) giving the nucleation rate is accelerated versus time due to the increase of difference $P_{i}(z, t)-P_{0}(z, t)$ in which $P_{\mathrm{i}}$ is more or less proportional to $P_{\mathrm{g}}$. A possible tentative model is equation (14) which depends strongly on fluence.

$$
P_{g}(z, t)=P_{g}(0, t) e^{-\alpha z}
$$

\subsection{Limits of prediction}

The approximations of the model should be recalled to estimate the future applicability of the results to other systems, especially to natural biopolymers like collagens which are good candidates for artificial type of cellular matrix materials. To obtain the pressure wave, the Grüeneisen function was used as a constant and equal to one for polymers. This has no incidence on the dynamics but only on the shape of the waveform. The nucleation rate is a product of exponential factor, Zeldovich frequency factor and molecular concentration factor $n_{0}$. All of them require slight approximations because $T$ and $P$ dependence of the parameters are most of the time not known. Among them viscosity $\eta$ and surface tension $\sigma$ play an important role. If viscosity dependence on $T$ and $P$ is known for some synthetic polymers like PVAc, it is not the case of surface tension and unfortunately biopolymers are not well documented with that respect. Also the molecular concentration factor $n_{0}$ has been tentatively attributed to the concentration of free volume holes of PVAc. Again biopolymers literature does not report such value of $n_{0}$ due to the more complex molecular structures of the biological systems. The growing importance of the new present field is hopefully going to boost new studies.

\section{Properties of the obtained polymer foams}

The laser-induced foams obtained so far have shown that the molecular structure of the polymer or biopolymer is conserved by the laser treatment which then appears as a very mild method for local processing. This has been proved by spectroscopic methods like Raman micro-spectrometry and fluorescence (Wiesniewski et al., 2007; Gaspard et al., 2008c). In most cases the post-treatment material has spectral signature similar to the starting polymers. This is true for a large range of fluence although for values much larger than the threshold slight traces of carbon particles can be detected on the foam samples. An appealing feature of this laser method to obtain foamy material is the high density of nucleation which can be evaluated at $1 \mu \mathrm{m}^{-3}$. Such high densities are not achieved by classical methods and confer to the final laser-induced polymer foam a large potential of new applications. The use of high values of laser fluence can achieve nano-structures like 
filaments in high concentration (Fig.5d). The obtained 3D network made of nano-sized filaments have valuable properties, for future experiments in biomedical applications like artificial cellular matrices for tissue repair, for chemical or biosensors, for gas or nanofiltration.

\section{Future experiments and applications}

Little is known on the part of target which is ejected from the surface during the development of the foam layer. However we are in the presence of a mechanism which does not require a high temperature but rather relies on the force field created by the pressure developed during the confinement. The surface material in a fluid state is accelerated non uniformely along the normal of the surface due to the cavitation phenomenon, stronger below the surface as indicated by the model. As a result an extensive fragmentation of this portion of polymer occurs and is sometime visible in the form of a small cloud of white smoke ejected at high speed from the laser spot. When the laser irradiation is done trough a silica window placed near the sample surface, this cloud of nanoparticles is deposited on its surface as seen in Fig.16. The experiment is performed in air and does not require a vacuum, and can therefore be easily adapted to the deposition of nanoparticles onto substrates that do not stand the vacuum, like cell cultures in biological research or other polymer science experiments. Similar results have been repeated for the polymers studied in this work. As suggested by Raman micro-spectrometry the chemical structure of the polymer is not destroyed by the laser treatment and the atomic force microscopy indicates that the nanodroplets are in a molten state when they hit the silica surface. This preparation of nanoparticles by laser ablation in the photomechanical regime is new and will probably open up further ideas of future applications.
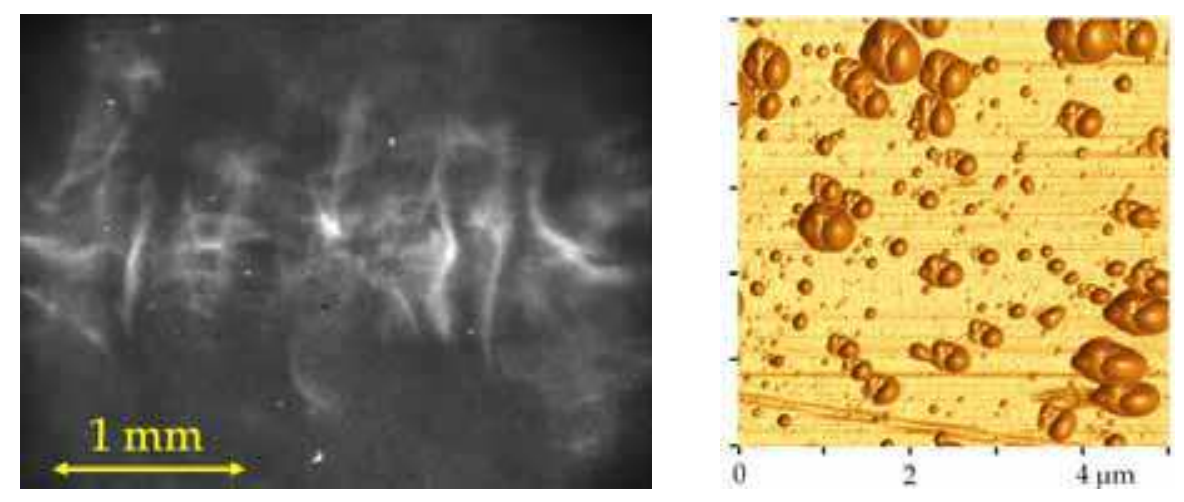

Fig. 16. Nanoparticles of gelatin deposited by $\mathrm{KrF}$ laser ablation in air $\left(F_{0}=1.7 \mathrm{~J} / \mathrm{cm}^{2}\right)$ on a silica window placed $300 \mu \mathrm{m}$ in front the target. Several square spots of $1 \mathrm{~mm}$ were used with a $1 \mathrm{~mm}$ shifting between each. (left) Optical microscope image with scattered light. (right) Atomic force microscope image.

The obtained foams are attractive substrates for building new cell culture media because of their interesting properties that are approaching that of the natural extracellular matrices. Based on the small numbers of already studied polymers it can be said that with a laser 
pulse of high fluence, several times the threshold fluence, the foam structure is forced to develop open cells configuration due to large amount of ablation gas formed. This means that the new material is permeable to fluids like in the natural tissue. However, due to the small characteristic dimensions of the laser-induced foam, usually in the nanometer or submicrometer range, the stability in the contact with liquids is a critical parameter which needs to be carefully studied. To circumvent this difficulty cross-linking the polymer before or after laser step may be an interesting treatment. The model has shown how the rate of cavitation is sensitive to viscosity which in turn can be modified by cross-linking reagents. There are some biopolymers or materials which are naturally cross-linked. For instance this is the case of keratin which is stable in liquids due to the significant amount of SS cross-links formed by dimerisation of cystein. Fig.17 is the illustration that two different synthetic polymers do not behave identically. CAP tends to give nano-droplets and CTA preferably forms nano-filaments for fluencies much larger than threshold. Future mechanistic investigation will bring more insight to explain these peculiarities.

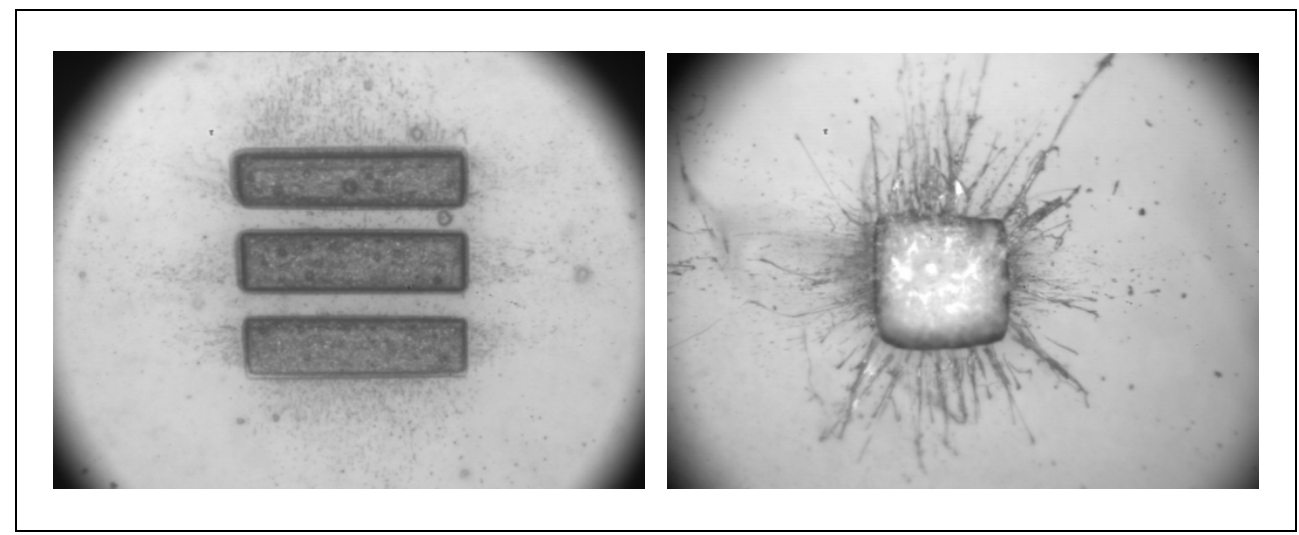

Fig. 17. KrF laser ablation with a single pulse on a $150 \mu \mathrm{m}$ spot. (left) CAP ablated at $F_{0}=1.5 \mathrm{~J} / \mathrm{cm}^{2}$; the spot is divided in three parts for the purpose of focusing. (right) CTA ablated at $8 \mathrm{~J} / \mathrm{cm}^{2}$ tends to produce many small filaments by ejection of molten polymer whereas lower fluence yields only foam.

\section{Conclusions}

A new type of polymer foam is obtained with the aid of laser ablation. Biopolymers and synthetic polymers concerned by the laser-induced foaming have absorption coefficient of the order of $10-10^{3} \mathrm{~cm}^{-1}$ in order to allow the pressure to increase during the radiation absorption by pressure confinement. So far the experiments have been performed with one pulse of ns lasers in the condition of pressure confinement and single fs pulses for which this condition is necessarily met and no polymer one-photon absorption is needed. The mechanisms of foaming are studied experimentally and by modelling with a set of several biopolymers. The dynamics of foam formation was monitored and showed an appearance time of the order of 50-100 ns after the start of the laser pulse. This characteristic time is explained by the necessity of the high pressure component of the laser-induced acoustic wave to propagate away from the absorption depth, a phenomenon which occurs the speed 
of sound, and to be reflected off at the target surface to launch a tension wave back to the bulk of the polymer. When the tension intensity rises and combines with the action of the ablation gases, it overpasses the critical tensile strength of the polymer. The phenomenon of cavitation occurs giving rise to the formation of critical bubbles which further grow, merge and stabilize to form a dense nanofoam. The proposed model of nucleation rate based on the classical theory of homogeneous nucleation accounts well for the experimental ns and fs laser dynamics. A new numerical development was possible for poly(vinyl acetate), a model synthetic polymer rich in literature data. The rate of nucleation is expressed in three factors which are each discussed and explicited numerically. The importance of the temperature and pressure dependence of the viscosity is revealed by the results. Also the model brings some information on the depth profile of the foaming kinetics which has a maximum below the surface at a well defined depth, e.g. $27 \mu \mathrm{m}$ for PVAc and the $\mathrm{KrF}$ laser. It is proposed that the holes of free volume, known in polymers by o-PALS and theoretical equation of state, can be at the inception of the critical nucleus involved in the cavitation step. The laserinduced modifications, foams with closed or open cells, filaments, ejected nano-droplets, etc have appealing properties and unprecedented by competing and more classical techniques. Many new and promising experiments are made possible by these results concerning lasermaterial microprocessing based on a photomechanical regime of ablation. Future detailed studies of the specificities of new polymers and biopolymers may open up more applications.

\section{References}

Angell, C.A. (1985). Spectroscopy simulation and scattering, and the medium range order problem in glass. J.Non-Cryst.Solids, Vol 73., No 1-3., (August 1985), (1-17), ISSN 00223093

Avramov, I. \& Milchev, A. (1988). Effect of disorder on diffusion and viscosity in condensed systems. J.Non-Cryst.Solids, Vol 104., No 2-3., (September 1988), (253-260), ISSN 00223093

Baidakov,V.G. (2007). Explosive Boiling of Superheated Cryogenic Liquids, WileyVCHSpringer, ISBN 3527405755, 9783527405756, Berlin, Germany.

Barus, C., (1891). The dependence of viscosity on pressure and temperature. Proc.Amer.Acad., Vol 27., (1892), (13)

Bäuerle, D. (2000). Laser Processing and Chemistry, 3rd edn. Springer, ISBN 978-3-540-668916, Berlin,. Heidelberg, Germany.

Budd, P.; McKeon, N.B. \& Fritsch, D. (2005). Free volume and intrinsic microporosity in polymers. J.Mat.Chem., Vol 15., No 20., (May 2005), (1977-1986), ISSN 09599428

Cohen, M.H. \& Turnbull, D. (1959). Molecular Transport in Liquid and Glasses. J.Chem.Phys., Vol 31., No 5., (November 1959), (1164-1169), ISSN 00219606

Dlubek, G.; Lüpke, T.; Stejny, J.; Alam, M. \& Arnold, M. (2000). Local Free Volume in Ethylene-Vinyl Acetate Copolymers: A Positron Lifetime Study. Macromol., Vol 33., No 3., (February 2000), (990-996), ISSN 00249297

Elaboudi, I.; Lazare, S.; Belin, C.; Talaga, D. \& Labrugère, C. (2008). Underwater excimer laser ablation of polymers. Appl.Phys.A, Vol 92., No 4., (September 2008), (743-748), ISSN 0947-8396 
Gandini, A. (2008). Polymers from Renewable Resources: A Chalenge for the future of Macromolecular Materials. Macromol., Vol 41., No 24., (December 2008), (94919504), ISSN 0024-9297

Gaspard, S.; Oujja, M.; DeNalda, R.; Abrusci, C.; Catalina, F.; Banares, L.; Lazare, S. \& Castillejo, M. (2007). Nanofoaming in the surface of biopolymers by femtosecond pulsed laser irradiation. Appl.Surf.Sci., Vol.254, No 4, (December 2007), (1179-1184) ISSN 01694332

Gaspard, S.; Oujja, M.; de Nalda, R.; Castillejo, M.; Bañares, S.; Lazare, S. \& Bonneau, R. (2008a). Nanofoaming dynamics in biopolymers by femtosecond laser irradiation. Appl.Phys.A, Vol.93, No.1, (October 2008), (209-213), ISSN 0947-8396

Gaspard, S.; Forster, M.; Huber, C.; Zafiu, C.; Trettenhahn, G.; Kautek, W. \& Castillejo, M. (2008b). Femtosecond laser processing of biopolymers at high repetition rate. Phys.Chem.Chem.Phys., Vol.10, No.40, (October 2008), (6174-6181), ISSN 14639076

Gaspard, S.; Oujja, M.; Abrusci, C.; Catalina, F.; Lazare, S.; Desvergne, J.P. \& Castillejo, M. (2008c). Laser induced foaming and chemical modifications of gelatine films. J.Photochem.Photobiol.A:Chem., Vol 193., No 2-3., (January 2008), (187-192), ISSN 10106030.

Gordon, M.K. \& Hahn, R.A. (2010). Collagens. Cell.Tissue Res., Vol.339, No.1, (247-257), ISSN 0302766X

Guggenheim, E.A. (1945). The principle of the corresponding states. J.Chem.Phys., Vol 13., No 7., (July1945), (253-261), ISSN 00219606

Hong-ling Lv; Bao-guo Wang \& Ji-chu Yang (2008). Correlations between PALS and diffusivities in amorphous polymer-solvent systems. Desalinisation, Vol.234 No.1-3, (December 2008), (33-41), ISSN 00119164

Kupcik, J.; Blasvska-Gilev, J. \& Pola, J. (2005). IR Laser-Induced Degradation of Poly(vinyl acetate): Novel Thermal Reactions in Solid. Macromol.Rap.Commun., Vol.26 No.5, (March 2005), (386-389), ISSN 10221336

Lazare, S. \& Tokarev, V. (2005a). Ultraviolet Laser Ablation of Polymers and the Role of Liquid Formation and Expulsion. In: Recent Advances in Laser Processing of Materials, Elsevier, ISBN 0750660791, Oxford England

Lazare, S.; Tokarev, V.; Sionkowska, A. \& Wisniewski, M. (2005b). Surface foaming of collagen, chitosan and other biopolymer films by $\mathrm{KrF}$ excimer laser ablation in the photomechanical regime. Appl.Phys.A, Vol 81., No 3., (May 2005), (465-470), ISSN 0947-8396

Lazare, S.; Bonneau, R.; Gaspard, S.; Oujja, M.; De Nalda, R.; Castillejo, M. \& Sionkowska, A.; (2009). Modeling the dynamics of one laser pulse surface nanofoaming of biopolymers. Appl.Phys.A, Vol 94., No 4., (March 2009), (719-729), ISSN 0947-8396

Lazare, S.; Elaboudi, I.; Castillejo M. \& Sionkowska, A., (2010). Model Properties relevant to Laser Ablation of moderately absorbing polymer. Appl.Phys.A, Vol 9X., No X., (Xxx 2010), (ppp-ppp), ISSN 0947-8396

Lennard-Jones, J. E. \& Devonshire, A. F. (1937), Critical Phenomena in Gases I, Proc.R.Soc.Lond. A, Vol.163, No.912, (November 1937), (53-70), ISSN 1364-5021

Lippert, T. \& Dickinson, J. T. (2003). Chemical and Spectroscopic Aspects of Polymer Ablation: Special Features and Novel Directions. Chem.Rev., Vol.103, No.2, (January 2004), (453-486), ISSN 0009-2665 
Liu, C-F; Sun, R-C; Zhang, A-P; Qin, M-H; Ren, J-L \& Wang, X-I, (2007). Preparation and Characterization of Phthalated Cellulose Derivatives in Room-Temperature Ionic Liquid without Catalysts. J.Agric.Food Chem., Vol. 55, No 6, (March 2007), (23992406), ISSN 00218561

McKinney, J.E. \& Belcher, H.V. (1963). Dynamic compressibility of poly(vinyl acetate) and ist relation to free. J.Res. oft he NIST, Vol.67A, No.1, (January-February 1963), (4353), ISSN 1044-677X

Mooney, B.P. (2009). The second green revolution? Production of plant-based biodegradable plastics. Biochem.J., Vol.418, No.2, (March 2009), (219-232), ISSN 02646021

Moore, C.J. (2008). Synthetic polymers in the marine environment: a rapidly increasing, long-term threat. Environ.Res., Vol 108, No.2, (Oct 2008), (131-139), ISSN 0013-9351

Pérez, J. ; Muñoz-Dorado, J. ; de la Robia, T. \& Martinez, J. (2002). Biodegradation and biological treatments of cellulose, hemicellulose and lignin: an overview, Int.Microbiolo., Vol.5, No.2 , (June 2002), (53-63), ISSN 1139-6709

Pinkert, A.; Marsh, K.N.; Pang, S. \& Staiger, M.P. (2009). Ionic liquids and their interaction with cellulose, Chem.Rev., Vol.109, No.12 , (December 2009), (6712-6728), ISSN 00092665

Moore, C.J. (2008). Synthetic polymers in the marine environment: a rapidly increasing, long-term threat. Environ.Res., Vol 108, No.2, (Oct 2008), (131-139), ISSN 0013-9351

Oujja, M. ; Pérez, S., Fadeeva, E. ; Koch, J.; Chichkov, B.N. \& Castillejo, M., (2009). Three dimensional microstructuring of biopolymers by femtosecond laser irradiation. Appl.Phys.Lett., Vol.95, No.26, (December 2009), (263703-3p), ISSN 00036951

Roland, C.M. \& Casalini, R., (2003). Temperature and volume effects on local segmental relaxation in poly(vinyl acetate). Macromol., Vol.36,No.4, (February 2003), (13611367), ISSN 00249297

Sionkowska, A. ; Wisniewski, M.; Lazare, S.; Lopez, J.; Hernandez, M-C., Guillemot, F. \& Durrieu, M-C. (2008). Surface properties of femtosecond laser irradiated collagen films. Mol.Cryst.Liq.Cryst., Vol.486, (January 2008), (250-256), ISSN 15421406

Telle, H.U. ; Urena, A.G. \& Donovan, R.J. (2007). Laser Chemistry, J Wiley \& Sons, ISBN 9780-48570-4 (HB), England

Tao, S.J. (1972). Positronium annihilation in molecular substances. J.Chem.Phys., Vol 72., No 11., (June 1972), (5499-5510), ISSN 00219606

Tokarev, V.; Lopez, J.; Lazare, S. \& Weisbuch, F. (2003). High aspect ratio microdrilling in polymers with UV laser ablation: experiment and model. Appl.Phys.A, Vol 76., No 3., (March 2003), (385-393), ISSN 0947-8396

Weisbuch, F.; Lazare, S.; Goodall, F.N. \& Débarre, D. (1999). Submicron-resolution ablation with a $\mathrm{KrF}$ excimer laser beam patterned with a projection. Appl.Phys.A, Vol 69., Suppl. No 1., (December 1999), (S413-S417), ISSN 0947-8396

Wendorf, J.H. \& Fisher, E.W. (1973). Thermal density fluctuations in amorphous polymers as revealed by small angle X-ray diffraction. Coll.EPol.Sci., Vol 251., No 11., (November 1973), (876-883), ISSN 0303-0402

Williams, M.L.; Landel, R.F. \& Ferry,D (1955). The temperature dependence of relaxation mechanisms in amorphous polymers and other glass-forming liquids. J.Amer.Chem.Soc., Vol 77., No 14., (1942), (3701-3707), ISSN 27863. 
Wisniewski, M.; Sionkowska, A.; Kaczmarek, H; Lazare, S.; Tokarev, V. \& Belin, C. (2007). Spectroscopic study of a $\mathrm{KrF}$ ecximer laser treated surface oft hin collagen films. J.Photochem.Photobiol.A:Chem., Vol 188., No 2-3., (May 2007), (192-199), ISSN 10106030.

Zeldovich, Y., (1942). Theory of Formation of New Phase, Cavitation. Sov.J.Exp.Theor.Phys., Vol 12., No 11., (1942), (525-538), ISSN 0044-4510 


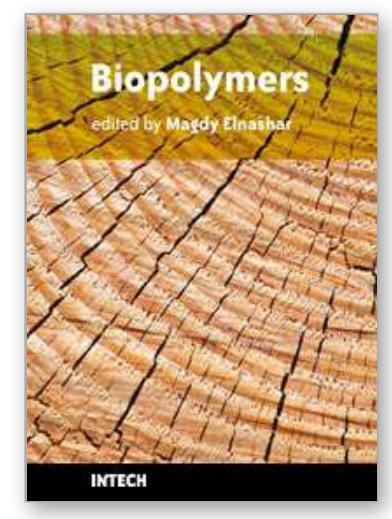

\author{
Biopolymers \\ Edited by Magdy Elnashar
}

ISBN 978-953-307-109-1

Hard cover, 612 pages

Publisher Sciyo

Published online 28, September, 2010

Published in print edition September, 2010

Biopolymers are polymers produced by living organisms. Cellulose, starch, chitin, proteins, peptides, DNA and RNA are all examples of biopolymers. This book comprehensively reviews and compiles information on biopolymers in 30 chapters. The book covers occurrence, synthesis, isolation and production, properties and applications, modification, and the relevant analysis methods to reveal the structures and properties of some biopolymers. This book will hopefully be of help to many scientists, physicians, pharmacists, engineers and other experts in a variety of disciplines, both academic and industrial. It may not only support research and development, but be suitable for teaching as well.

\title{
How to reference
}

In order to correctly reference this scholarly work, feel free to copy and paste the following:

Sylvain Lazare (2010). Microfoams of Biopolymers by Laser-Induced Stretching: Mechanisms and Applications, Biopolymers, Magdy Elnashar (Ed.), ISBN: 978-953-307-109-1, InTech, Available from: http://www.intechopen.com/books/biopolymers/microfoams-of-biopolymers-by-laser-induced-stretchingmechanisms-and-applications

\section{INTECH}

open science | open minds

\section{InTech Europe}

University Campus STeP Ri Slavka Krautzeka 83/A 51000 Rijeka, Croatia Phone: +385 (51) 770447

Fax: +385 (51) 686166 www.intechopen.com

\section{InTech China}

Unit 405, Office Block, Hotel Equatorial Shanghai No.65, Yan An Road (West), Shanghai, 200040, China 中国上海市延安西路65号上海国际贵都大饭店办公楼405单元 Phone: +86-21-62489820

Fax: +86-21-62489821 
(C) 2010 The Author(s). Licensee IntechOpen. This chapter is distributed under the terms of the Creative Commons Attribution-NonCommercialShareAlike-3.0 License, which permits use, distribution and reproduction for non-commercial purposes, provided the original is properly cited and derivative works building on this content are distributed under the same license. 